\title{
Double radiative pion capture on hydrogen and deuterium and the nucleon's pion cloud
}

\author{
S. Tripathi ${ }^{1}$, D.S. Armstrong ${ }^{2}$, M.E. Christy ${ }^{1}$, J.H.D. Clark, ${ }^{4 \ddagger}$, T.P. Gorringe ${ }^{1}$, M.D. \\ Hasinoff $^{3}$, M.A. Kovash ${ }^{1}$, D.H. Wright, ${ }^{3 \dagger}$, and P.A. Żołnierczuk ${ }^{1}$. \\ ${ }^{1}$ Department of Physics and Astronomy, University of Kentucky, \\ Lexington, KY, USA 40506. \\ 2 Department of Physics, College of William and Mary, \\ Williamsburg, VA, USA $2318 \%$. \\ ${ }^{3}$ Department of Physics and Astronomy, University of British Columbia, \\ Vancouver, BC, Canada V6T $1 Z 1$.
}

(September 8, 2018)

\begin{abstract}
We report measurements of double radiative capture in pionic hydrogen and pionic deuterium. The measurements were performed with the RMC spectrometer at the TRIUMF cyclotron by recording photon pairs from pion stops in liquid hydrogen and deuterium targets. We obtained absolute branching ratios of $(3.02 \pm 0.27$ (stat. $) \pm 0.31$ (syst. $)) \times 10^{-5}$ for hydrogen and $\left(1.42 \pm_{0.12}^{0.09}\right.$ (stat. $) \pm 0.11$ (syst. $\left.)\right) \times 10^{-5}$ for deuterium, and relative branching ratios of double radiative capture to single radiative capture of $(7.68 \pm 0.69$ (stat. $) \pm 0.79($ syst. $)) \times 10^{-5}$ for hydrogen and $\left(5.44 \pm_{0.46}^{0.34}\right.$ (stat. $) \pm$ 0.42 (syst. $)) \times 10^{-5}$ for deuterium. For hydrogen, the measured branching ratio and photon energy-angle distributions are in fair agreement with a reaction mechanism involving the annihilation of the incident $\pi^{-}$on the $\pi^{+}$cloud of the target proton. For deuterium, the measured branching ratio and energyangle distributions are qualitatively consistent with simple arguments for the expected role of the spectator neutron. A comparison between our hydrogen and deuterium data and earlier beryllium and carbon data reveals substantial changes in the relative branching ratios and the energy-angle distributions and is in agreement with the expected evolution of the reaction dynamics from an annihilation process in S-state capture to a bremsstrahlung process in P-state capture. Lastly, we comment on the relevance of the double radiative process to the investigation of the charged pion polarizability and the in-medium pion field.
\end{abstract}




\section{INTRODUCTION}

A cornerstone of quantum chromodynamics at low energies is the pion's emergence as the approximate Goldstone boson of a spontaneously broken chiral symmetry. Consequently, the pion is central to understanding the realization of QCD symmetries and their associated currents in low energy hadronic processes. In particular, the emission and absorption of pions by nucleons is closely connected to the low energy manifestation of the partially conserved axial current.

Radiative pion capture, $\pi N \rightarrow \gamma N$, and pion photo-production, $\gamma N \rightarrow$ $\pi N$, are well-known benchmarks for experimental tests of the low energy realization of approximate chiral symmetry. The chiral predictions for pion photo-production and radiative pion capture were originally obtained from current algebra techniques [1] and are nowadays derived from heavy baryon chiral perturbation theory $[2,3]$. Such arguments relate the threshold amplitude for absorption or emission of s-wave pions to the axial currents of the nucleon and the pion. The resulting relationship for $\gamma p \rightarrow \pi^{+} n$ photoproduction - the Kroll-Ruderman theorem and higher-order corrections - is experimentally verified to a few-percent accuracy.

Less well known - both theoretically and experimentally - is double radiative pion capture, $\pi N \rightarrow \gamma \gamma N$, and radiative pion photo-production, $\gamma N \rightarrow \gamma \pi N$. Nonetheless the absorption or emission of pions in double radiative capture or radiative photo-production is similarly constrained by chiral symmetry. Predictions for double radiative pion capture $\pi^{-} p \rightarrow \gamma \gamma n$ have been derived from a current algebra approach by Lapidus and Muskhanov [4] and a chiral Lagrangian approach by Beder $[5,6]$ and Cammarata [7]. Most interestingly, all studies have suggested that threshold absorption from $\ell=0$ orbitals involves the annihilation of the incident $\pi^{-}$on the $\pi^{+}$cloud of the target proton, the double radiative process thus exhibiting intriguing sensitivity to the nucleon's pion cloud in the small-to-moderate $q^{2}$ regime.

Originally, some excitement was generated by the prediction of $\pi^{-} \pi^{+} \rightarrow$ $\gamma \gamma$ annihilation in double radiative capture. The context was speculations on pion condensation or precursor effects in ordinary nuclei, which motivated Ericson and Wilkin [8], Nyman and Rho [9], and Barshay [10] to advance the $(\pi, 2 \gamma)$ reaction on nuclear targets as a selective probe of the pion field in the nuclear medium. While pion condensation at nuclear densities is nowadays unimaginable, the investigation of the pion field in the nuclear medium is still of continuing importance.

Recently, new studies [11] have attempted to determine the pion's electromagnetic polarizability via radiative pion photo-production. The method involves the Compton scattering of the incident $\gamma$-ray from the $\pi^{+}$cloud of the target proton - i.e. the proton acting as a pion target - with the polarizability being encoded in the amplitude for the $\gamma \pi \rightarrow \gamma \pi$ scattering. Consequently, a better understanding of the pion cloud contribution to $\gamma \pi \rightarrow \gamma \pi$ scattering

in radiative photo-production or $\pi \pi \rightarrow \gamma \gamma$ annihilation in double radiative 
capture is of some importance.

Unfortunately, the predicted branching ratios for double radiative capture in pionic atoms are small - of order of $10^{-4}$ - and the experimental work is extremely challenging. Nevertheless, experiments by Deutsch et al. at CERN [12] and Mazzucato et al. at TRIUMF [13] were successful in recording gamma-ray pairs from double radiative capture on ${ }^{9} \mathrm{Be}$ and ${ }^{12} \mathrm{C}$ targets. However, the elementary process of double radiative pion capture on hydrogen, and the simplest nuclear process of double radiative pion capture on deuterium, have so far remained unobserved. ${ }^{1}$

Herein we report the results of experimental work on double radiative capture in pionic hydrogen and pionic deuterium. The experiment was conducted with the RMC spectrometer at the TRIUMF cyclotron and detected photon-pairs from pion stops in liquid hydrogen and deuterium targets. Our results include absolute branching ratios, relative branching ratios, and photon energy-angle distributions for double radiative capture on hydrogen and deuterium. Our goals in conducting the measurements were to: test the predictions for the reaction dynamics of the elementary process, compare the results for the elementary process and the simplest nuclear process, and examine the evolution of double radiative capture from $Z=1$ to complex nuclei. A letter on our experimental results for the elementary process has been previously published [15]. ${ }^{2}$

This article is organized as follows. In Sec. II we briefly outline the theoretical aspects of the $\pi N \rightarrow \gamma \gamma N$ process. In Sec. III we discuss the background difficulties arising from real $\gamma$-ray coincidences and accidental $\gamma$-ray coincidences from pion charge exchange, single radiative capture and other processes. Sec. IV describes the experimental setup and trigger electronics. The identification of the double radiative capture events, subtraction of the two-photon background events, and determination of the two-photon detection efficiency are described in Sec. V. In Sec. VI we discuss our results for hydrogen and deuterium, and compare our experimental data for these elementary processes with earlier data for the nuclear process. Our conclusions are presented in Sec. VII.

\footnotetext{
${ }^{1} \mathrm{~A}$ branching ratio upper limit $\leq 5.5 \times 10^{-4}$ for double radiative capture on pionic hydrogen was obtained by Vasilevsky et al. [14].

${ }^{2}$ In addition, in Refs. [16,17] we conducted searches for exotic baryon and dibaryon resonances by detection of photon-pairs following pion capture in hydrogen and deuterium.
} 


\section{THEORETICAL OVERVIEW}

\section{A. Initial state}

Pionic atoms are initially formed in atomic orbitals with large principal quantum numbers $(n)$ and an approximately statistical population of the orbital sub-states $(\ell)$. The atoms then de-excite by a combination of Auger transitions and radiative transitions with the cascade ultimately terminating in the nuclear absorption process (for details see Ref. [18]).

In pionic hydrogen and pionic deuterium the nuclear absorption is dominated by S-state capture from $n=3-6$ orbitals [19-22]. This unique feature of $Z=1$ capture originates from the combination of strong Stark mixing between degenerate $\ell$-orbitals and weak $\ell>0$ nuclear absorption and is firmly established by the available pionic x-ray and strong interaction data [19-23].

By comparison, in pionic beryllium and pionic carbon the nuclear capture is predominantly from P-states. For $\pi^{-12} \mathrm{C}$, Sapp et al. [24] have given summed absorption probabilities of $0.08 \pm 0.03$ for S-state capture, $0.92 \pm 0.03$ for P-state capture, and $\sim 7 \times 10^{-4}$ for D-state capture. For $\pi^{-9} \mathrm{Be}$, Sapp et al. [24] have tabulated x-ray yields that imply summed absorption probabilities of $\sim 0.2$ for S-state capture and $\sim 0.8$ for P-state capture.

\section{B. Reaction kinematics}

For the $\pi^{-} p \rightarrow \gamma \gamma n$ elementary process the important kinematical quantities are shown in Fig. 1. We denote the pion 4-momentum $\pi^{\mu}=\left(m_{\pi}, 0\right)$, the proton 4-momentum $p^{\mu}=\left(M_{p}, 0\right)$, the neutron 4-momentum $n^{\mu}=\left(E_{n}, \overrightarrow{p_{n}}\right)$, and the photon 4 -momenta as $k_{1}^{\mu}=\left(\omega_{1}, \overrightarrow{k_{1}}\right)$ and $k_{2}^{\mu}=\left(\omega_{2}, \overrightarrow{k_{2}}\right)$. The pion, proton and neutron masses are $m_{\pi}, M_{p}$ and $M_{n}$ respectively.

The measured quantities in our experiment are the two photon energies $\omega_{1}, \omega_{2}$ and the photon-pair opening angle cosine, $y=\cos \theta$. In terms of measured quantities the 3-momentum transfer $\vec{q}=\overrightarrow{p_{n}}$ and energy transfer $q_{o}=E_{n}-M_{p}$ are

$$
|\vec{q}|=\sqrt{\omega_{1}^{2}+\omega_{2}^{2}+2 \omega_{1} \omega_{2} y}
$$

and

$$
q_{o}=m_{\pi}-\omega_{1}-\omega_{2}
$$

where the binding energy of the pionic atom has been neglected. Note that the 4-momentum transfer-squared $q^{2}$ is space-like and ranges from zero, for back-to-back photons with equal energy partition, to $-m_{\pi}^{2}$, for either parallel photons or a photon energy at the end-point energy.

In the $\pi^{-} p \rightarrow \gamma \gamma n$ elementary process the photon energies range from 0 to $129.4 \mathrm{MeV}$ and the neutron kinetic energy ranges from 0 to $8.9 \mathrm{MeV}$. The $\gamma \gamma n$ 3-body phase space distribution strongly favors the equal sharing of the available energy between the two photons and somewhat favors large photon-pair opening angles over small photon-pair opening angles. 


\section{Reaction dynamics}

Calculations of the $\pi^{-} p \rightarrow \gamma \gamma n$ elementary process have been performed by Joseph [25], Lapidus and Musakanov [4] and Beder [5] for at-rest capture and Beder [6] and Cammarata [7] for in-flight capture. For at-rest capture on hydrogen the predicted absolute branching ratios are $5.1 \times 10^{-5}$ [25], $5.1 \times 10^{-5}$ [4] and $5.4 \times 10^{-5}[5]$, and the corresponding ratios $R(2 \gamma / 1 \gamma)$ of double radiative capture to single radiative capture are $1.4 \times 10^{-4}, 1.4 \times 10^{-4}$ and $1.3 \times 10^{-4}$, respectively.

At tree-level the $\pi N \gamma$ effective Lagrangian of Beder [5] implies three categories of contributions to double radiative capture: the $\pi \pi$ diagrams of Fig. 2.a-b, with both photons attached to the pion, the NN diagrams of Fig. 2.c-e, with both photons attached to the nucleon, and the $\pi N$ diagrams of Fig. 2.f-g, with one photon attached to the pion and one photon attached to the nucleon. The graphs in Fig. 2.a-b and 2.f-g are leading order $O(1)$, and the graphs in Fig. 2.c-e are next-to-leading order $O\left(1 / M_{n}\right)$, in the modern terminology of heavy-baryon chiral perturbation theory [28].

In threshold $\ell=0$ capture $\left(e . g .{ }^{1} \mathrm{H}\right.$ and $\left.{ }^{2} \mathrm{H}\right)$ the contributions of diagrams 2.b and 2.f-g vanish and the only surviving leading-order graph is the four-point $\pi \pi$ annihilation graph of Fig. 2.a. The four-point $\pi \pi$ annihilation diagram yields a squared matrix element with kinematical dependence [5]

$$
|M . E .|^{2} \propto\left(\omega_{1}^{2}+\omega_{2}^{2}+2 \omega_{1} \omega_{2} y\right)\left(1+y^{2}\right) /\left(m_{\pi}\left(\omega_{1}+\omega_{2}\right)-\omega_{1} \omega_{2}(1-y)\right)^{2}
$$

that peaks at small opening angles and equal energy partition. This sole $O(1)$ contribution to $\ell=0$ capture, involving annihilation of the stopped $\pi^{-}$on the $\pi^{+}$-field of the target proton, and offering sensitivity to the nucleon's pion cloud and the $\pi \pi \rightarrow \gamma \gamma$ vertex, is the most intriguing feature of the double radiative process.

In addition, the NN diagrams of 2.c-e introduce a next-to-leading order $O\left(1 / M_{n}\right)$ contribution to threshold $\ell=0$ capture. These graphs yield a squared matrix element with kinematical dependence [5]

$$
|M . E .|^{2} \propto(1-y)^{2}
$$

that peaks at large opening angles but is independent of the energy partition between the two photons. According to Beder [5], the $O(1) \pi \pi$ graph 2.a contributes roughly $65 \%$, the $O\left(1 / M_{n}\right)$ NN graphs 2.c-e contribute roughly $20 \%$, and their interference terms contribute roughly $15 \%$, to the $\ell=0$ total branching ratio.

In threshold $\ell=1$ capture $\left(e . g .{ }^{9} \mathrm{Be}\right.$ and ${ }^{12} \mathrm{C}$ ) the leading order $\pi N$ diagrams 2.f-g are found to dominate, with small contributions from the $O(1) \pi \pi$ graphs and negligible contributions from the $O\left(1 / M_{n}\right) N N$ graphs. The $\pi N$ bremsstrahlung graphs 2.f-g yield a squared matrix element with kinematical dependence [5]

$$
|M . E .|^{2} \propto 1 / \omega_{1}^{2}+1 / \omega_{2}^{2}+\left(1+y^{2}\right) /\left(2 \omega_{1} \omega_{2}\right)
$$


that diverges for small photon energies. A striking difference between $\ell=0$ capture and $\ell=1$ capture is the prediction of a roughly ten-fold increase in the ratio $R(2 \gamma / 1 \gamma)$.

In addition to the above contributions from the $\pi N \gamma$ effective Lagrangian, the possible effects of virtual $\pi^{o}$ 's and intermediate $\Delta$ 's have been investigated by several authors $[4,8,10]$. The contribution of virtual $\pi^{o}$ 's, which involves virtual $\pi^{\circ \star} \rightarrow \gamma \gamma$ decay following off-shell $\pi^{-} p \rightarrow \pi^{\circ \star} n$ charge exchange, was found by Lapidus and Musakanov [4], Ericson and Wilkin [8] and Barshay [10] to be entirely negligible. The contribution of virtual $\Delta$ 's, which involve $\Delta$ excitation by pion absorption and $\Delta$ decay by photon emission, was estimated by Beder [5] to be roughly $5 \%$ in the $\ell=0$ process.

\section{Medium effects}

Of course, in double radiative capture on nuclear targets the dynamics of the elementary process must be folded with the nuclear response. Although no calculation for double radiative capture on deuterium has been performed a number of studies of double radiative capture on light nuclei have been published. The most detailed treatments of nuclear double radiative capture were published by Christillin and Ericson [26] and Gil and Oset [27].

Christillin and Ericson [26] combined an effective interaction incorporating both $\pi \pi$ and $\pi \mathrm{N}$ graphs with a phenomenological nuclear resonance incorporating the giant dipole and other resonances to calculate ${ }^{12} \mathrm{C}$ double radiative capture. They found that an important effect of the nuclear medium was a substantial quenching of the partial rate in the kinematical region $\left(\overrightarrow{k_{1}}+\overrightarrow{k_{2}}\right) \rightarrow 0$ due to Pauli blocking. Gil and Oset [27] combined an effective interaction that includes the $\pi \pi$ graphs but omits $\pi \mathrm{N}$ graphs with the Fermi gas model and the local density approximation to calculate ${ }^{9} \mathrm{Be}$ and ${ }^{12} \mathrm{C}$ double radiative capture. In contrast to Christillin and Ericson they found a significant enhancement of the partial rate in the kinematical region

$\left(\overrightarrow{k_{1}}+\overrightarrow{k_{2}}\right) \rightarrow 0$, and commented that medium modification of the pion field has a large effect for these kinematics. Note that both calculations indicated a large increase in the ratio $\mathrm{R}(2 \gamma / 1 \gamma)$ for the nuclear process over the elementary process.

\section{EXPERIMENTAL CHALLENGES}

The measured values of the branching ratios for the known modes of pion absorption on hydrogen and deuterium are listed in Table I. For hydrogen the capture modes are pion charge exchange, $\pi^{-} p \rightarrow \pi^{o} n$, single radiative capture, $\pi^{-} p \rightarrow \gamma n$, and radiative pair production, $\pi^{-} p \rightarrow e^{+} e^{-} n$ [29-31]. For deuterium the capture modes are non-radiative capture $\pi^{-} d \rightarrow n n$, radiative capture $\pi^{-} d \rightarrow \gamma n n$, and pion charge exchange $\pi^{-} d \rightarrow \pi^{o} n n$ [32,33]. By comparison the anticipated branching ratios for double radiative capture on 
hydrogen and deuterium are of order $10^{-4}$ - the tiny branching ratios, neutral particle final-states and continuum energy-angle distributions together making detection quite difficult. Moreover, these difficulties are magnified by intense backgrounds from real coincidences and accidental coincidences of photon pairs.

Neutral pion decay $\pi^{o} \rightarrow \gamma \gamma$ following at-rest pion charge exchange yields real $\gamma-\gamma$ coincidences, the $\pi^{o}$ recoil energy of $2.9 \mathrm{MeV}$ in $\pi^{-} p \rightarrow \pi^{o} n$ and $0-1 \mathrm{MeV}$ in $\pi^{-} d \rightarrow \pi^{o} n n$ imparting a Doppler broadening on the laboratory energy-angle distributions of the $\pi^{o} \rightarrow \gamma \gamma$ photons (the two-photon background from in-flight charge exchange was negligible compared to atrest charge exchange). In hydrogen the broadening yields an energy spread $55.0<\omega<83.0 \mathrm{MeV}$ and an angular spread $-1<y<-0.91$. In deuterium the broadening yields an energy spread $61.6<\omega<73.9 \mathrm{MeV}$ and an angular spread $-1<y<-0.97$. The $\pi^{o} \rightarrow \gamma \gamma$ background therefore prohibits a measurement of double radiative capture at large opening angles and endangers the measurement at small opening angles due to the finite resolution of the detector system. Since the branching ratio for charge exchange is much larger in hydrogen $(0.607 \pm 0.004[29])$ than deuterium $\left((1.45 \pm 0.19) \times 10^{-4}[32]\right)$ the $\pi^{o} \rightarrow \gamma \gamma$ background is more worrisome in hydrogen than in deuterium. Herein we denote the real $\gamma-\gamma$ coincidences from $\pi^{o} \rightarrow \gamma \gamma$ decay as the " $\pi^{o}$ background".

Charge exchange and single radiative capture yield photon-pairs via accidental $\gamma \gamma$ coincidences. In our experiment the incident beam had a microstructure with a pulse period of 43 ns and a pulse duration of 2-4 ns. Consequently, the probability of two pions stops in one beam pulse was not negligible. Such multiple pion stops can generate accidental $\gamma-\gamma$ coincidences via the detection of one photon from one pion stop and another photon from another pion stop in the same beam pulse. These accidental $\gamma-\gamma$ coincidences may comprise a pair of photons that originate from: two charge exchange events, two single radiative capture events, or one charge exchange event and one single radiative capture event. Such accidental $\gamma-\gamma$ coincidences have summed energies $106<\omega<258 \mathrm{MeV}$ and opening angles $-1<y<+1$. Herein we denote the accidental $\gamma-\gamma$ coincidences from multiple pion stops in one beam pulse as the " $2 \pi$ background".

Lastly, two possible sources of two-photon backgrounds were (i) Dalitz decay following pion charge exchange and (ii) $\mu^{-}$decay from in-beam muon contamination. In background process (i) the decay $\pi^{o} \rightarrow \gamma e^{+} e^{-}$can yield a photon-pair by identification of one true photon (the Dalitz-decay $\gamma$-ray) and one fake photon (the Dalitz-decay $e^{+} e^{-}$pair). In background process (ii) the $\mu^{-}$decay yields single photons via internal bremsstrahlung or external bremsstrahlung and photon pairs by accidental coincidences with other photons from pion stops. Although both backgrounds are much rarer than the $\pi^{o}$ background or the $2 \pi$ background, they are less straightforwardly differentiated from the double radiative capture events by the applied cuts (see Sec. V A). Herein we denote background (i) as the Dalitz background and 
background (ii) as the $\mu \pi$ background.

\section{EXPERIMENTAL SETUP}

Our measurements of $\left(\pi^{-}, 2 \gamma\right)$ capture were conducted with the RMC detector on the M9A beamline at the TRIUMF cyclotron. The RMC detector is a photon-pair spectrometer with large solid angle coverage and moderate energy resolution. The detector was developed for studies of radiative muon capture on hydrogen and nuclei, and has been discussed elsewhere [34,35].

The M9A beamline [36] provided a negative pion flux of typically $7 \times$ $10^{5} \mathrm{~s}^{-1}$ with a central momentum $81.5 \mathrm{MeV} / c$, momentum bite $9 \%$, and spot size $3.4 \times 4.4 \mathrm{~cm}^{2}$. The beam had a microscopic time structure consisting of a 2-4 ns pulse width with a 43.3 ns pulse separation and a $99 \%$ macroscopic duty cycle. For the hydrogen measurement we conducted the experiment with the M9A radio frequency separator which yielded a $\pi / e / \mu$ beam composition of $0.73 / 0.18 / 0.09$. For the deuterium measurement we conducted the experiment without the separator which yielded a $\pi / e / \mu$ beam composition of $0.06 / 0.89 / 0.05 .^{3}$

The incident beam particles were counted in a 4-element plastic scintillator telescope with a total thickness $0.635 \mathrm{~cm}$ and stopped in either a liquid hydrogen target or a liquid deuterium target. Both targets had cylindrical cross sections with dome-shaped front faces, and were located at the geometrical center of the detector system. The $\mathrm{H}_{2}$ cell was $15 \mathrm{~cm}$ in length, $16 \mathrm{~cm}$ in diameter, and $2.7 \ell$ in volume, with $\mathrm{Au}$ front and side walls of thickness $0.25 \mathrm{~mm}$. The $\mathrm{D}_{2}$ cell was $15 \mathrm{~cm}$ in length, $15 \mathrm{~cm}$ in diameter, and $2.5 \ell$ in volume, with $\mathrm{Cu}$ front and side walls of thickness $0.50 \mathrm{~mm}$. For the $\mathrm{H}_{2}$ experiment the hydrogen filling had a deuterium contamination of order $10^{-6}$ and for the $\mathrm{D}_{2}$ experiment the deuterium filling had a hydrogen contamination of order $10^{-3}$.

The outgoing photons were detected by pair conversion in a cylindrical $\mathrm{Pb}$ converter and $e^{+}-\mathrm{e}^{-}$tracking in two cylindrical tracking chambers. The $\mathrm{Pb}$ converter had a thickness $1.12 \pm 0.01 \mathrm{~mm}$, radius $13 \mathrm{~cm}$, and length $35 \mathrm{~cm}$, and was centered at the geometrical center of the detector system. The tracking chambers comprised a low mass cylindrical multi-wire proportional chamber and a large volume cylindrical drift chamber. The MWPC had an inner radius $26.5 \mathrm{~cm}$, outer radius $27.5 \mathrm{~cm}$, and active length $\sim 75 \mathrm{~cm}$, and consisted of 768 axial anode wires between $2 \times 384$ helical inner and outer cathode strips. It provided a single $(x, y, z)$ coordinate for each track. The drift chamber had an inner radius $30.2 \mathrm{~cm}$, outer radius $58.0 \mathrm{~cm}$ and active length $\sim 90 \mathrm{~cm}$, and comprised four concentric layers of drift cells with six anode wires per drift cell. Layers one, two and four were axial and provided $(x, y)$-coordinate tracking

\footnotetext{
${ }^{3}$ This running condition resulted from the failure of the RF separator in the $\mathrm{D}_{2}$ experiment.
} 
information. Layer three was stereo and provided the $z$-coordinate tracking information. The entire detector system was located in a large solenoidal magnet that provided a $1.2 \mathrm{kG}$ axial magnetic field.

Concentric rings of segmented plastic scintillators were employed for fasttriggering. These scintillators comprised: the $\mathrm{A} / \mathrm{A}^{\prime}$-rings, located just inside the $\mathrm{Pb}$ converter radius, the $\mathrm{C}$-ring, located just outside the $\mathrm{Pb}$ converter radius, and the $\mathrm{D}$-ring, located just outside the drift chamber radius. The A/A'-rings had four-fold segmentation and inner radii of 12.2 and $12.6 \mathrm{~cm}$ respectively. They were used to veto any charged particles that emerged from the vicinity of the target. The C-ring had twelve-fold segmentation and an inner radius $15.8 \mathrm{~cm}$. The $\mathrm{C}$ scintillators were used to identify the $e^{+} e^{-}$pairs from $\gamma$-ray conversion in the lead converter. The D-ring had sixteen-fold segmentation and an inner radius $\sim 59.4 \mathrm{~cm}$. The D scintillators were used to identify the individual electron and positron tracks that emerged from the drift chamber.

The drift chamber times were recorded by LeCroy 1879 pipeline TDCs while the MWPC hits were recorded by custom-built electronics [37]. The pulse height and timing information from the beam counters and trigger scintillators were recorded by CAMAC ADCs and TDCs. The data acquisition was based on two SLAC scanner processors (SSPs) [38] and a VAX 3000 workstation. One SSP handled the event readout from the hardware to an internal memory, the other SSP handled the data transfer from the internal memory to the computer, while the computer handled the data storage, run control, online monitoring, etc.

\section{A. Trigger system}

The two-photon trigger was based upon the hit multiplicities and hit topologies in both the trigger scintillator rings and the drift chamber cells. The trigger was designed for efficient identification of photon pairs from double radiative capture and efficient rejection of single photons and coincident photons from single radiative capture and pion charge exchange. The trigger was implemented in four stages that involved: trigger scintillator multiplicities (level one), trigger scintillator topologies (level two), drift chamber cell multiplicities (level three), and drift chamber cell topologies (level four).

Level one was based on the multiplicities of the hits in the trigger scintillators. The level-one condition was $\bar{A} \cdot \overline{A^{\prime}} \cdot \geq 2 C \cdot \geq 3 D$, where $\bar{A} \cdot \overline{A^{\prime}}$ imposed a veto on charged particles, $\geq 2 C$ required a pair of $\gamma$-ray conversions, and $\geq 3 D$ required a minimum of three tracks to fully traverse the drift chamber.

Level two was based on the topologies of the hits in the C-D trigger scintillators rings and was implemented using a LeCroy 2372 memory look-up unit (MLU) and a LeCroy 4508 programmable logic unit (PLU). First the MLU, using the recorded distribution of $\mathrm{C}$-counters hits, defined the allowed distribution of D-counter hits that was consistent with the topology of a double radiative capture event. Then the PLU, using the allowed distribution of D- 
counter hits, determined the number of so-called "valid Ds", i.e. the number of matches between allowed D-counters and fired D-counters. A total of $\geq 2$ non-adjacent C-counter hits were required to define an allowed distribution of D-counter hits, and a total of $\geq 3$ valid Ds were required to fulfill the level two trigger requirement.

Note that two different versions of the level two trigger were implemented for the hydrogen experiment and the deuterium experiment. In the hydrogen experiment, if two C-counters were back-to-back, i.e. were located on the opposite sides of the C-ring, no allowed distribution of D-counter hits was defined. This permitted a reduction in the trigger rate due to photon pairs from $\pi^{o} \rightarrow \gamma \gamma$ decay following $\pi^{-} p \rightarrow \pi^{o} n$ charge exchange. In the deuterium experiment, the branching ratio for charge exchange is small and therefore rejection of back-to-back C-counters was unnecessary.

Level three was based on the multiplicities of the cell hits in the drift chamber and was derived by custom-built OR boards [39], which indicated if a

cell was hit, and a custom-built analog hit counter [40], which accumulated the distribution of hits. The trigger imposed minimum values on the number of the individual cell hits in the second drift chamber layer (denoted $n_{2}$ ) and the number of contiguous cell clusters in the third and fourth drift chamber layers (denoted $n_{3-4}$ ). The values of $n_{2}$ and $n_{3-4}$ were chosen for good efficiency in accepting photon-pair events and good efficiency in rejecting single photon events. In the hydrogen measurement we collected some data (45\%) with $\left(n_{2}, n_{3-4}\right) \geq(3,3)$ and some data $(55 \%)$ with $\left(n_{2}, n_{3-4}\right) \geq(4,6)$. In the deuterium experiment we collected all data with $\left(n_{2}, n_{3-4}\right) \geq(4,6)$.

Level four was based on the topologies of the cell hits in the first layer of the drift chamber. This trigger was implemented in software by the SSP and was based on cell information from the OR boards. It rejected any event with back-to-back cell hits in drift chamber layer one and was useful in the reduction of the event rate from $\pi^{o} \rightarrow \gamma \gamma$ decay following $\pi^{-} p \rightarrow \pi^{o} n$ charge exchange. The level four trigger was enabled for the hydrogen measurement but disabled for the deuterium measurement

A dedicated $\pi^{o}$ trigger - which allowed the back-to-back topologies of $\gamma$-ray conversions from $\pi^{o}$ decays - was used for measurements of the spectrometer acceptance, resolution function, etc. A dedicated beam trigger which generated events from pre-scaled hits in beam counters - was used for the determination of the pion content of the incident beam.

\section{DATA ANALYSIS.}

We report hydrogen and deuterium data that were collected in two fourweek running periods. The hydrogen experiment recorded a total of $3.10 \times 10^{11}$ pion stops and the deuterium experiment recorded a total of $3.07 \times 10^{11}$ pion stops.

Note that the running conditions for data taking in hydrogen and deuterium were different in several ways. Firstly a separated beam was used in the 
hydrogen experiment, while an unseparated beam was used in the deuterium experiment (see Sec. IV). For deuterium this resulted in a large electron beam rate which therefore required greater attention to counting the $\pi^{-}$stops. Secondly the MWPC was not operable at the large electron beam rate of the deuterium experiment. For deuterium this resulted in less z-tracking information and poorer z-tracking resolution. Thirdly, the level two trigger and the level four trigger were different in rejecting back-to-back hit topologies for the hydrogen experiment but accepting back-to-back hit topologies for the deuterium experiment.

The data analysis involved several steps. In step one we applied cuts to identify photon pairs that originated from the target. In step two we applied cuts to select the photon pairs from double radiative capture and reject the photon pairs from various background sources. In step three we determined the contributions of any residual background events in the double radiative capture events. Lastly, we determined the number of negative pion stops, and obtained the photon pair acceptance, in order to derive the branching ratio for double radiative capture.

\section{A. Identification of photon pairs}

The analysis procedure involved first identifying the various tracks in the tracking chambers, then pairing the $e^{+} e^{-}$tracks to make individual photons, and finally pairing the individual photons to make photon pairs. The criteria for identifying "good tracks" and making "good photons" are described below.

\section{Tracking cuts}

Each helical track is defined by a circle fit to the $(x, y)$ coordinates and a straight-line fit to the $(\rho, z)$ coordinates that were obtained from the chamber hits. The tracks fell into two categories: so-called "non-wrap around" tracks where the particle emerges through the drift chamber outer radius, and so-called "wrap around" tracks where the particle spirals within the drift chamber outer radius. We imposed requirements on the maximum variance $\left(S_{x y}^{2}, S_{z}^{2}\right)$ and the minimum points $\left(N_{x y}, N_{z}\right)$ in the $(x, y)$ circle fit and $(\rho, z)$ straight-line fit. In addition, for hydrogen, a requirement was imposed on the maximum distance $d_{w c}$ of the particle's trajectory from the associated MWPC hit.

The cut parameters for the hydrogen analysis and the deuterium analysis are summarized in Table II. Note that the $(x, y)$ resolution is somewhat poorer for positrons than electrons - due to the $2.7^{\circ}$ Lorentz angle of the drift electrons in the $1.2 \mathrm{kG}$ magnetic field - and therefore the values of $S_{x y}^{2}$ are larger for $e^{+}$tracks. For hydrogen a cut was also imposed on the distance $d_{w c}$ between the fitted track and the corresponding MWPC hit. 


\section{Photon cuts}

The $e^{+} e^{-}$tracks from $\gamma \rightarrow e^{+} e^{-}$conversion must converge at the $\mathrm{Pb}$ converter radius. Therefore in pairing the $e^{+} e^{-}$tracks into reconstructed photons we imposed a maximum on the $\mathrm{x}, \mathrm{y}$ separation $\left(d_{x y}\right)$ and the $\mathrm{z}$ separation $\left(d_{z}\right)$ of the $e^{+} e^{-}$tracks at the $\mathrm{Pb}$ radius. In addition, we required that the reconstructed photon must have originated from the direction of the target cell. Therefore a cut was imposed on the magnitude $\left(r_{\text {close }}\right)$ and the z-component $\left(z_{\text {close }}\right)$ of the closest approach of the photon momentum vector to the detector's geometrical center

The values of cuts on $d_{x y}, d_{z}, r_{\text {close }}$ and $z_{\text {close }}$ for hydrogen and deuterium are summarized in Table III. Note that the z-tracking was poorer in the deuterium experiment than the hydrogen experiment, due to the inoperable MWPC. This is reflected in the different values for $d_{z}$ and $z_{\text {close }}$ in hydrogen and deuterium.

\section{Photon pair events}

Good photon pairs were defined as events with four good tracks, i.e. four tracks passing all tracking cuts in Table II, and two good photons, i.e. two photons passing all photon cuts in Table III. From $3.10 \times 10^{11}$ pion stops in hydrogen a total of $2.3 \times 10^{6}$ photon pairs survived the combination of tracking cuts and photon cuts. From $3.07 \times 10^{11}$ pion stops in deuterium a total of $2.3 \times 10^{5}$ photon pairs survived the combination of tracking cuts and photon cuts.

The summed energy $\left(\omega_{1}+\omega_{2}\right)$ spectra, individual energy $(\omega)$ spectra, and opening angle cosine $(y)$ spectra, for reconstructed photon pairs from hydrogen and deuterium, are shown in Fig. 3. The photon energies $\omega$ were obtained via the sum of the $e^{+} e^{-}$momentum vectors at the converter radius, i.e. $\vec{p}_{\gamma}=\vec{p}_{e^{+}}+\vec{p}_{e^{-}}$, and the opening angle $y$ was obtained via the scalar product of the two $\gamma$-ray momenta, i.e. $y=\vec{p}_{\gamma 1} \cdot \vec{p}_{\gamma 2} /\left|\vec{p}_{\gamma 1}\right|\left|\vec{p}_{\gamma 2}\right|$. Note that the $e^{+}, e^{-}$transverse momenta $p_{t}$ were derived from the track radius $r$ in the circle fit via $p_{t}=e r B$, and the $e^{+} e^{-}$total momentum $p$ was derived using the track pitch $\phi$ from the straight-line fit via $p=p_{t} / \sin \phi$.

The spectra in Fig. 3 are dominated by real photon coincidences from the $\pi^{o}$ background and accidental photon coincidences from the $2 \pi$ background. The $\pi^{o}$ background is most readily seen in the opening angle spectra as events with $y<-0.7$ and the $2 \pi$ background is most readily seen in the sum energy spectra as events with $E>150 \mathrm{MeV}$. The different numbers of photon pairs from ${ }^{1} \mathrm{H}$ and ${ }^{2} \mathrm{H}$ - i.e. $2.3 \times 10^{6}$ versus $2.3 \times 10^{5}$ - reflects the much larger $\pi^{o}$ background in the ${ }^{1} \mathrm{H}$ experiment than in the ${ }^{2} \mathrm{H}$ experiment. 


\section{B. Identification of double radiative capture events}

Next we describe the cuts we applied on the beam counter pulse height and C-counter timing information to remove the $2 \pi$ and $\mu \pi$ backgrounds and the opening angle to remove the $\pi^{o}$ background. These cuts are denoted the beam counter amplitude cut, the $\mathrm{C}$-counter timing cut and the opening angle cut, respectively.

\section{Beam counter amplitude cut}

To distinguish between single pion stops and multiple pion stops we applied a cut on the pulse height information from the four beam counters. First, the pulse heights from the eight photo-multipliers viewing the four beam scintillators were normalized and summed to obtain a single beam counter amplitude. The resulting amplitude spectra from representative ${ }^{1} \mathrm{H}$ and ${ }^{2} \mathrm{H}$ runs are shown in Fig. 4. The lower energy peak corresponds to events with single $\pi^{-}$ stops while the higher energy peak corresponds to events with two $\pi^{-}$stops. In the ${ }^{1} \mathrm{H}$ spectrum the peak widths were dominated by the energy straggling of the incoming pions and the finite resolution of the beam counters. In the ${ }^{2} \mathrm{H}$ spectrum the peak widths were further increased by additional pile-up with beam electrons.

Also indicated in Fig. 4 are the corresponding beam counter amplitude cuts at Chan. 1069 in hydrogen and Chan. 1150 in deuterium. The cut efficiencies for passing two-photon events from single $\pi^{-}$stops were determined utilizing the photon pairs from $\pi^{o} \rightarrow \gamma \gamma$ decay via both dedicated $\pi^{o}$ trigger runs and standard two-photon trigger runs. We found an efficiency of $100 \%$ for the hydrogen data and $92 \%$ for the deuterium data.

\section{C counter timing cut}

Two categories of accidental $\gamma \gamma$ coincidences are capable of circumventing the beam counter amplitude cut. They are: (i) $\mu \pi$ background events involving one prompt $\gamma$-ray from a $\pi^{-}$stop and one delayed $\gamma$-ray from an earlier $\mu^{-}$ stop, and (ii) $\pi \pi$ background events originating from two separate $\pi^{-}$stops in two adjacent beam pulses. Note that the $\mu \pi$ background was much larger in the unseparated-beam deuterium experiment than in the separated-beam hydrogen experiment.

To remove such backgrounds we applied a $\mathrm{C}$ counter timing cut. The cut was based on the time difference $\left(t_{C 1}-t_{C 2}\right)$ between the two C-counters that were fired by the two $e^{+} e^{-}$pairs in the two-photon event. For hydrogen we used a timing window of $-4<\left(t_{C 1}-t_{C 2}\right)<+4 \mathrm{~ns}$ and for deuterium we used a timing window of $-5<\left(t_{C 1}-t_{C 2}\right)<+7$ ns (the $t_{C 1}-t_{C 2}$ time difference spectrum for deuterium photon pairs is shown in Fig. 5). Their corresponding efficiencies for passing real $\gamma \gamma$ coincidences were determined 
utilizing the photon pairs from $\pi^{o} \rightarrow \gamma \gamma$ decay using both dedicated $\pi^{o}$ trigger runs and standard two-photon trigger runs. We found an efficiency of $99.5 \%$ for the hydrogen data and $99.0 \%$ for the deuterium data.

\section{Opening angle cut}

Finally, we applied a cut on the opening angle cosine $y$ to remove the $\pi^{o}$ background (the distributions in opening angle for all photon-pairs from hydrogen and deuterium are shown in Fig. 3). In our final analyzes we used values of $y \geq-0.1$ for hydrogen and deuterium although studies were made of the sensitivity of our results to the cut value. Note that (i) the $\pi^{o}$ background was much larger in the hydrogen measurement and (ii) the opening angle resolution was significantly poorer in the deuterium measurement.

The efficiency of the opening cut for passing events from double radiative capture is obviously dependent on the angular distribution for the $(\pi, \gamma \gamma)$ events. The cut's effect on $(\pi, \gamma \gamma)$ events is discussed in detail in Sec. VI.

\section{Double radiative capture events}

The resulting distributions of events in individual energy, summed energy and opening angle - after the tracking, photon, beam counter amplitude, $\mathrm{C}$ counter timing and opening angle cuts - are shown in Fig. 6 for hydrogen and deuterium. For hydrogen the spectra contain a total of 665 two-photon events with 597 events having sum energies $<150 \mathrm{MeV}$ and 566 events having sum energies $80-150 \mathrm{MeV}$ (the sum energy cut $<150 \mathrm{MeV}$ removes the vast majority of the $\pi \pi$ background events and the sum energy cut $>80 \mathrm{MeV}$ removes the vast majority of any Dalitz background events). For deuterium the spectra contain a total of 521 two-photon events with 335 events having sum energies $<150 \mathrm{MeV}$ and 327 events having sum energies $80-150 \mathrm{MeV}$.

The summed photon energy spectrum shows a peak at $E \sim m_{\pi}$ that corresponds to the double radiative capture events. ${ }^{4}$ The photon pairs with summed energies $>150 \mathrm{MeV}$ are dominated by accidental $\gamma \gamma$ coincidences from unrejected $\pi \pi$ background. Note that the energy distribution of the accidental $\gamma \gamma$ coincidences are different in hydrogen and deuterium - the former being dominated by gamma-rays following $\left(\pi^{-}, \pi^{o}\right)$ charge exchange, the latter being dominated by gamma-rays following $\left(\pi^{-}, \gamma\right)$ radiative capture. The individual photon energy spectrum shows a broad continuum with a low energy cut-off at about $20 \mathrm{MeV}$ from the acceptance of the spectrometer. At individual photon energies $>100 \mathrm{MeV}$ the spectra are also dominated by the accidental $\gamma \gamma$ coincidences from the $\pi \pi$ background.

\footnotetext{
${ }^{4}$ The peak centroid is shifted downwards due to energy loss of the $e^{+} e^{-}$pairs in traversing the lead converter, trigger scintillators, etc.
} 
A typical double radiative capture event is shown in Fig. 7. It has zero hits in the A-counter ring, two hits in the C-counter ring, and four hits in the D-counter ring. The topology of trigger scintillator hits and drift cell hits is consistent with diverging $e^{+} e^{-}$pairs, and therefore the event fulfilled the two-photon trigger.

\section{Subtraction of background events}

A small fraction of $\pi^{o}$ background events and $\pi \pi$ background events were found to survive the beam amplitude, C-counter timing and opening angle cuts - and therefore to contaminate the sum energy region $<150 \mathrm{MeV}$ of double radiative capture events. Below we describe the methods we used to subtract these backgrounds.

\section{1. $\pi^{o}$ background}

To estimate the number of $\pi^{o}$ background events that survived the cuts we utilized (i) the measured opening angle distribution for $\pi^{o}$ events at angles $y<-0.45$ and (ii) the simulated opening angle spectrum for $\pi^{o}$ events at all angles.

The Monte Carlo simulation was performed using the CERN GEANT3 package [41] (see Sec. V E for details). Recall, the true angular distributions of $\gamma-\gamma$ coincidences from $\pi^{o}$ decay following at-rest charge exchange on hydrogen are limited to $-1.0<y<-0.91$ (the $\pi^{o}$ 's originating from charge exchange on hydrogen dominate both the hydrogen data-set and the deuterium dataset). However, the measured angular distribution of $\gamma-\gamma$ coincidences from $\pi^{o}$ decay shows a significant opening angle tail from the pair spectrometer response function.

Fig. 8 compares the measured and simulated opening angle spectra for the region $-1.0<y<-0.6$ using a dedicated $\pi^{o}$ trigger (Sec. IV A). It indicates good agreement between measurement and simulation for $\pi^{\circ}$ events, and affirms our confidence in using the simulation to determine the angular response of the pair spectrometer and to estimate the $\pi^{o}$ contamination in the region $y>-0.1$.

Fig. 8 also compares the angular distribution from measurement and simulation for the region $-0.8<y<+1.0$ using the standard $2 \gamma$ trigger (Sec. IV A) for the photon-pairs surviving all the applied cuts except the angle cut. The simulated data in Fig. 8 imply a small fraction of $\pi^{\circ}$ events with reconstructed opening angles $y>-0.1$ - thus contaminating the region of double radiative capture events. To estimate the $\pi^{o}$ contamination we defined two windows: a $\pi^{o}$ window of $-0.7<y<-0.45$ in which the $\pi^{o}$ background is dominant, and a $(\pi, 2 \gamma)$ window of $-0.1<y<+1.0$ in which the $(\pi, 2 \gamma)$ signal is dominant. Using these windows the $\pi^{o}$ contamination with $y>-0.1$ was determined via 


$$
N_{2 \gamma}^{E X}=N_{\pi^{o}}^{E X}\left(N_{2 \gamma}^{M C} / N_{\pi^{o}}^{M C}\right)
$$

where $N_{\pi^{o}}^{E X}$ is the number of measured events in the $\pi^{o}$ window and $N_{2 \gamma}^{M C}$ and $N_{\pi^{o}}^{M C}$ are the number of simulated events in the $\pi^{o}$ and $(\pi, 2 \gamma)$ windows. For hydrogen the procedure yielded a $\pi^{O}$ background estimate of $53 \pm 30$ events. and for deuterium the procedure yielded a $\pi^{o}$ background estimate of $2 \pm 1$ events. Note that the errors in the estimate of the $\pi^{o}$ background are dominated by the statistical uncertainties in the $\pi^{O}$ simulation. The simulation was based on generating a total of $9 \times 10^{7} \pi^{o}$ decays from at-rest charge exchange.

\section{2. $\pi \pi$ background}

To estimate the number of $\pi \pi$ background events that survived the cuts we utilized their measured sum energy spectra in Fig. 9. The summed energy spectra were obtained by selecting the photon pairs failing the beam counter amplitude cut. In the hydrogen spectrum (Fig. 9 solid curve), the lower energy peak at $\sim 120 \mathrm{MeV}$ is predominantly two $\gamma$-rays from two $\pi^{o} \rightarrow \gamma \gamma$ decays while the higher energy peak at $\sim 180 \mathrm{MeV}$ is predominantly one $\gamma$-ray from $\pi^{o} \rightarrow \gamma \gamma$ decay and another $\gamma$-ray from single radiative capture. In the deuterium spectrum (Fig. 9 dashed curve), the single broad peak at $\sim 230 \mathrm{MeV}$ originates from two $\gamma$-rays from two single radiative capture events.

Clearly the sum energy of $\pi \pi$ events can exceed the kinematical limit for double radiative capture events. We therefore defined an upper threshold $E_{\circ}$, above which double radiative capture events are absent but $\pi \pi$ background events are present, and computed the number of $\pi \pi$ events in the double radiative capture region $<150 \mathrm{MeV}$ from number of $\pi \pi$ events in the high energy region $>E_{\circ}$. For hydrogen this method gave $48 \pm 8 \pi \pi$ background events below $150 \mathrm{MeV}$ in the double radiative capture sum energy spectrum of Fig. 6. For deuterium the same method gave $20 \pm 2 \pi \pi$ background events below $150 \mathrm{MeV}$ in the double radiative capture sum energy spectrum of Fig. 6 . The total number of $\pi \pi$ events at all energies were $\sim 100$ in hydrogen and $\sim 200$ in deuterium.

A slight complication in this approach was the finite resolution of the pair spectrometer. This generates a small high energy tail of double radiative capture events that extends beyond their kinematic limit. We therefore varied the upper threshold $E_{\circ}$ from 160 to $180 \mathrm{MeV}$ to examine the sensitivity to effects of the high energy tail of the spectrometer resolution function. For both hydrogen and deuterium the resulting variations in the resulting estimates of the $\pi \pi$ contamination were smaller than $1 \sigma$. 


\section{Other backgrounds}

One potential source of background events was accidental coincidences between a delayed photon from $\mu$ decay and a prompt photon from $\pi$ capture - the $\mu \pi$ background. Such events were present in the unseparated-beam deuterium measurement but absent in the separated-beam hydrogen measurement. In deuterium, the contamination from $\mu \pi$ events that survived the C-counter timing cut was estimated from the $\mu \pi$ continuum background in the $t_{C 1}-t_{C 2}$ time spectrum. This procedure yielded a background estimate of $(15 \pm 4) \mu \pi$ events in the deuterium data set.

Another potential source of background events was $\pi^{o} \rightarrow \gamma e^{+} e^{-}$Dalitz decay following $\pi^{-} p \rightarrow \pi^{o} n$ charge exchange with the combination of the Dalitz pair and the $\gamma$ conversion yielding a photon-pair event. Although the branching ratio for the $\pi^{o} \rightarrow \gamma e^{+} e^{-}$decay mode is small $(1.2 \%)$ and the inefficiency of the $A, A^{\prime}$ veto is tiny $\left(<1 \times 10^{-4}\right)$, it is possible for Dalitz events to generate photon pairs with small opening angles. We therefore conducted a GEANT3 simulation of Dalitz events which showed that the Dalitz events with small opening angles $(y>-0.1)$ had small summed energies $(<80 \mathrm{MeV})$. Consequently, a worst-case estimate of the Dalitz background in the sum energy range $80-150 \mathrm{MeV}$ was made by attributing all events with $<80 \mathrm{MeV}$, to Dalitz background, giving a worst-case contribution of $<2$ events in hydrogen and $<1$ event in deuterium.

Another potential source of background events is nuclear double radiative capture from pion stops in neighboring materials. Using (i) the fraction (15 \pm 1)\% of pion stops in materials other than liquid $\mathrm{H}_{2} / \mathrm{D}_{2}$ (see Sec. VI), and (ii) an estimated branching ratio $5 \times 10^{-6}$ for nuclear double radiative capture with $\mathrm{E}>25 \mathrm{MeV}$ and $y>-0.1[12,13]$, we derived upper limits of $<10$ nuclear $(\pi, 2 \gamma)$ events in the hydrogen data-set and $<20$ nuclear $(\pi, 2 \gamma)$ events for the deuterium data-set. In addition, the fiducial cuts on photon trajectories will likely remove a large fraction of such events from pion stops in the target walls, beam scintillators, trigger scintillators, etc. We therefore treated the above estimates as very conservative upper limits on the nuclear $(\pi, 2 \gamma)$ background.

\section{Background-subtracted events.}

A compilation of the various estimates of the background sources in the hydrogen experiment and the deuterium experiment are given in Table IV. After subtracting the backgrounds, we obtained a total of $465 \pm_{40}^{39}$ double radiative capture events with summed energies from 80 to $150 \mathrm{MeV}$ in hydrogen and a total of $292 \pm_{27}^{19}$ double radiative capture events with summed 
energies from 80 to $150 \mathrm{MeV}$ in deuterium. ${ }^{5}$ The quoted uncertainties include the statistical errors and the systematic errors in the signal counts and the background estimates.

\section{Counting of incident pions}

The determination of the total number of incident pions was made by counting the total number of incident beam particles and measuring the pion fraction of the incident beam. The latter measurement was performed utilizing the beam trigger (Sec. IV A) and resulting amplitude and timing information from the beam counters. Note that the beam trigger events were collected simultaneously with the $2 \gamma$ trigger events for the duration of the measurements.

Because of the $43 \mathrm{~ns}$ micro-time structure of the primary proton beams the arrival of secondary pions, muons and electrons are separated in time. At $81.5 \mathrm{MeV} / \mathrm{c}$ the FWHM of pion arrival times was roughly $4.6 \mathrm{~ns}$, while the separation from electron and muon arrivals was $\sim 14$ and $\sim 21 \mathrm{~ns}$, respectively. In addition, the different particles have different energy loss and therefore different pulse heights in the beam counters. The combination of arrival time and pulse height at the beam counters was sufficient to clearly differentiate between incoming particles in both the separated beam and the unseparated beam. A representative plot of arrival time versus pulse height for deuterium - showing the quality of separation between pions, muons and electrons - is shown in Fig. 10.

Based on the pulse height and time discrimination between particle types we determined an average beam composition for the hydrogen experiment of $73 \% \pi^{-}, 18 \% e^{-}$and $9 \% \mu^{-}$and the deuterium experiment of $6 \% \pi^{-}, 89 \% e^{-}$ and $5 \% \mu^{-}$. These pion fractions were used to derive the number of livetimecorrected incident pions in the hydrogen experiment of $(3.10 \pm 0.03) \times 10^{11}$ and the deuterium experiment of $(3.07 \pm 0.15) \times 10^{11}$. The quoted uncertainties are based on the analysis of the pion fraction in the incident beam and are larger for the unseparated-beam deuterium experiment than for the separated-beam hydrogen experiment.

\section{E. Acceptance of pair spectrometer}

To compare the experimental data to theoretical calculations one requires a determination of the two-photon response of the detector system. The response function represents the probability of reconstructing photon pairs

\footnotetext{
${ }^{5}$ Note that in Ref. [15] we quote $482 \pm 42$ as the number of double radiative capture events with summed energies $>80 \mathrm{MeV}$ rather than $465 \pm_{40}^{39}$ events with summed energies $80-150 \mathrm{MeV}$. However, the data set is identical.
} 
with true energies $\omega_{1}, \omega_{2}$ and opening angle $y$ with measured energies $\omega_{1}^{\prime}$, $\omega_{2}^{\prime}$ and opening angle $y^{\prime}$, thus yielding the measured distribution of photon pairs from the true distribution of photon pairs. The response includes the detector geometry, the interaction and conversion of photons, the energy loss and multiple scattering of electrons, and the effects of the hardware trigger and the software cuts.

To determine the detector response function we employed a Monte Carlo simulation (RMCGEANT) that was based on the CERN GEANT3 package [41]. It incorporated the full geometry of the detector system - i.e. beam counters, target assembly, trigger counters, tracking chambers and the surrounding magnet - and all relevant interactions of photons and electrons. The simulation produced data files containing Monte Carlo events that were analyzed with the same software and the same cuts as the measured data. The program included event generators for pion charge exchange $\pi^{-} p \rightarrow \pi^{o} n$, single radiative capture $\pi^{-} p \rightarrow \gamma n$ and double radiative capture $\pi^{-} p \rightarrow \gamma \gamma n$. The generator we used for double radiative capture was based on formulas contained in Beder's article [5].

An important test of the GEANT simulation of the detector system was afforded by the photon pairs from the $\pi \pi$ background. These pairs originate from separate $\pi^{-} p \rightarrow \gamma n$ and/or $\pi^{-} p \rightarrow \pi^{0} n$ reactions from two pion stops in one beam pulse. Since the absolute yields and energy-angle distributions of such pairs are well known, they offer an invaluable means of calibration across the full range of photon energies and opening angles. In the hydrogen experiment this calibration procedure was performed continuously for the entire duration of the data taking. In the deuterium experiment the procedure was limited to dedicated calibration runs with liquid $\mathrm{H}_{2}$ filling. ${ }^{6}$

Representative measured and simulated spectra for photon-pairs from $\pi \pi$ events are compared in Fig. 11. The energy-angle distributions from experiment and simulation are in agreement at the level of a few percent and affirms the high quality of the GEANT simulation of the detector system. Note that the absolute efficiency for detecting photon pairs was found to be smaller in the experiment than the simulation by a factor $F=0.90 \pm 0.09$ in hydrogen and $F=0.96 \pm 0.06$ in deuterium. This difference is believed to originate from detector inefficiencies that are present in the experiment but are absent from the simulation. These inefficiencies include trigger scintillator inefficiencies, tracking chamber inefficiencies, and possible effects from chamber noise on track recognition. For both hydrogen and deuterium the run-by-run variation of the normalization factor was $\pm 4 \%$ and the total change over the running period was $<10 \%$.

\footnotetext{
${ }^{6}$ During running on deuterium the random $\gamma-\gamma$ coincidences from the $\pi \pi$ background and true $\gamma-\gamma$ coincidences from the $\pi^{o}$ background were used to monitor the relative acceptance for photon pairs. An absolute determination of the acceptance was not possible due to uncertainties in the hydrogen contamination in the liquid deuterium.
} 
Another important test of the overall quality of the GEANT simulation of the detector system was afforded by photon pairs from $\pi^{o} \rightarrow \gamma \gamma$ decay following $\pi^{-} p \rightarrow \pi^{o} n$ charge exchange. These data were collected in runs using a dedicated $\pi^{o}$ trigger in both the hydrogen experiment and the deuterium experiment. Likewise the measured data and simulated data for photon pairs from $\pi^{o} \rightarrow \gamma \gamma$ decay show good agreement and this also affirms the high quality of the GEANT simulation of the detection system. The normalization factors of $F=0.90$ in hydrogen and $F=0.96$ in deuterium that were derived from the $\pi \pi$ study are also consistent with the $\pi^{o}$ study.

The detector acceptance $\epsilon \Delta \Omega$ for double radiative capture was obtained using the RMCGEANT package and the double radiative capture event generator. The procedure yielded acceptances of $\epsilon \Omega=0.66 \times 10^{-4}$ for hydrogen and $\epsilon \Omega=0.90 \times 10^{-4}$ for deuterium when using the standard energy-angle cuts and assuming photon-pairs with energy-angle distributions derived by Beder [5]. Note that the acceptance for deuterium is larger than hydrogen due to the looser cuts that were applied in the presence of the smaller $\pi^{o}$ background.

\section{RESULTS}

Herein we discuss the experimental results from the hydrogen experiment and the deuterium experiment. We first describe the determination of the branching ratios in the two experiments and then discuss the comparison between theory and experiment for the elementary process and the nuclear process.

\section{A. Branching ratios for hydrogen and deuterium}

The absolute branching ratios for double radiative capture on pionic hydrogen and pionic deuterium were obtained via

$$
B . R .=\frac{N_{\gamma \gamma}}{N_{\pi^{-}} \cdot \epsilon \Delta \Omega \cdot F \cdot c_{s t o p} \cdot c_{b m}}
$$

where $N_{\gamma \gamma}$ is the number of background-subtracted double radiative capture events, $N_{\pi^{-}}$is the number of livetime-corrected incident pions, $\epsilon \Delta \Omega \cdot F$ is the detector acceptance, and $c_{\text {stop }}$ and $c_{b m}$ are minor correction factors which are discussed below.

The values employed in computing the branching ratios for hydrogen and deuterium are summarized in Table V. For the double radiative capture events with sum energies $80<\omega_{1}+\omega_{2}<150 \mathrm{MeV}$ and opening angles $y>-0.1$ we took $N_{\gamma \gamma}=465 \pm_{40}^{39}$ for hydrogen and $N_{\gamma \gamma}=292 \pm_{27}^{19}$ for deuterium from Sec. $\mathrm{V} \mathrm{C} 4$. For the livetime-corrected pion stops we took $N_{\pi}=(3.10 \pm 0.03) \times 10^{11}$ for hydrogen and $N_{\pi}=(3.07 \pm 0.15) \times 10^{11}$ for deuterium from Sec. VD. The GEANT simulation of detector acceptances of Sec. VE gave $\epsilon \Delta \Omega=$ 
$0.66 \times 10^{-4}$ for hydrogen and $\epsilon \Delta \Omega=0.90 \times 10^{-4}$ for deuterium. The factors $F=0.90 \pm 0.09$ for hydrogen and $F=0.96 \pm 0.06$ for deuterium account for small differences between the measured and the calculated acceptances due to various detector efficiencies, etc. The factor $c_{\text {stop }}=0.85 \pm 0.01$ accounts for the fraction of incident pions stopping in liquid hydrogen or liquid deuterium and was computed via a Monte Carlo simulation [34]. The factor $c_{b m}=0.98$ for hydrogen and $c_{b m}=0.91$ for deuterium accounts for efficiencies of events surviving the beam telescope amplitude and C-counter timing cuts that are omitted from the simulation (the uncertainties in $c_{b m}$ are negligible).

Using these values and Eqn. 7 we obtained absolute branching ratios of $(3.02 \pm 0.27$ (stat.) \pm 0.31 (syst.) $) \times 10^{-5}$ for hydrogen and $\left(1.42 \pm_{0.12}^{0.09}\right.$ (stat.) \pm 0.11 (syst.) $) \times 10^{-5}$ for deuterium. The corresponding values of ratios $R(1 \gamma / 2 \gamma)$ of double radiative capture to single radiative capture are $(7.68 \pm 0.69$ (stat. $) \pm$ 0.79 (syst.) $) \times 10^{-5}$ for hydrogen and $\left(5.44 \pm_{0.46}^{0.34}\right.$ (stat.) \pm 0.42 (syst. $\left.)\right) \times 10^{-5}$ for deuterium. The quoted uncertainties are dominated by the statistical error in the number of photon pairs and the systematic error in the acceptance for double radiative capture events.

For completeness we also quote the partial widths $\Gamma$ for double radiative capture. We use the recent measurements of the total widths of the $1 \mathrm{~S}$ atomic states of $0.86 \pm 0.07 \mathrm{eV}$ in pionic hydrogen (Schroeder et al. [42]) and $1.13 \pm 0.13 \mathrm{eV}$ in pionic deuterium (Hauser et al. [43]). Combined with our absolute branching ratios they yield partial widths of $\Gamma_{1 S}=26.0 \pm 4.1 \mu \mathrm{eV}$ for hydrogen and $\Gamma_{1 S}=16.2 \pm 2.5 \mu \mathrm{eV}$ for deuterium.

The sensitivity of the branching ratios to the opening angle cut and the sum energy cut are given in Table VI. Variations of the opening angle cut from $-0.2 \geq y \geq 0.0$ changed the branching ratio by $7 \%$ in hydrogen and $2 \%$ in deuterium. Variation of sum energy minimum from 60 to $80 \mathrm{MeV}$ and sum energy maximum from 150 to $170 \mathrm{MeV}$ changed the branching ratios by $5 \%$ in hydrogen and $7 \%$ in deuterium. A separate analysis of the two hydrogen data-sets with the different level-three triggers (Sec. IV A) gave branching ratios that differed by $7 \%$. In all cases the variations are significantly smaller than the quoted errors on the branching ratios.

We stress that the quoted branching ratios are for photon pairs with all possible angles and all possible energies. Their derivation assumes the energyangle distributions of photon pairs calculated by Beder [5] - the calculation and measurement being consistent for the visible region of the angle spectra $y>-0.1$ and energy spectra $\omega>25 \mathrm{MeV}$.

To test the sensitivity to the energy-angle distribution of the photon pairs we also extracted branching ratios with two other energy-angle distributions. Assuming an energy-angle distribution given by only $\pi \pi$ annihilation graphs the resulting branching ratios are decreased by $13 \%$ in hydrogen and $14 \%$ in deuterium, the decrease reflecting the larger proportion of photon pairs with small opening angles. Assuming an energy-angle distribution given by threebody $\gamma \gamma \mathrm{n}$ phase space the resulting branching ratios are increased by $5 \%$ in hydrogen and $6 \%$ in deuterium, the increase reflecting the smaller proportion 
of photon pairs with small opening angles.

\section{B. Discussion of results for hydrogen}

Our measured absolute branching ratio $(3.02 \pm 0.27$ (stat.) \pm 0.31 (syst.) $) \times$ $10^{-5}$ for double radiative capture on hydrogen is in fair agreement with the predicted values of $5.1 \times 10^{-5}$ (Joseph [25]), $5.1 \times 10^{-5}$ (Lapidus and Musakhanov [4]) and $5.4 \times 10^{-5}$ (Beder [5]) that embrace the dominance of the $\pi \pi \rightarrow \gamma \gamma$ annihilation mechanism. The measured branching ratio is definitely grossly under-estimated by either an $\ell=0 \mathrm{NN}$ mechanism or an $\ell=0$ $\pi \mathrm{N}$ mechanism and definitely grossly over-estimated by an $\ell=1 \pi \mathrm{N}$ mechanism (see Sec. II C and Ref. [5] for details). Intriguingly, the measurement is very close to the prediction $3.5 \times 10^{-5}$ of the $O(1) \pi \pi$ mechanism in the absence of the $O\left(1 / M_{n}\right)$ NN correction (we offer no rationale for the omission of the $O\left(1 / M_{n}\right)$ corrections from the NN graphs).

A comparison between the theoretical predictions and the experimental data for the two-photon opening angle distribution is shown in Fig. 12. Indicated are the results of the full $\ell=0$ calculation, $O(1) \pi \pi$ contribution only and $O\left(1 / M_{n}\right)$ NN contribution only. Note that the theoretical curves have been convoluted with the response function of the detector system and incorporate such effects as $e^{ \pm}$energy loss and multiple scattering (see Sec. VE for details). The measured spectra for opening angles $y>-0.1$ are reasonably consistent with the angular dependences of both the full calculation and the $O(1) \pi \pi$ term only. The experimental data are not consistent with the angular dependence of the NN mechanism which falls too steeply with increasing $y$.

We consider the experimental results for the absolute branching ratio and the opening angle distribution to be in support of the underlying theoretical idea of a dominant $\pi \pi \rightarrow \gamma \gamma$ mechanism in the $\ell=0$ elementary process. However, the measured branching ratio of $3.02 \pm 0.27$ (stat.) \pm 0.31 (syst.) $\times 10^{-5}$ and calculated branching ratio of $5.1-5.4 \times 10^{-5}$ do differ by roughly $40 \%$. For radiative pion photoproduction the complete set of leading order (LO), nextto-leading order (NLO) and next-to-next-to-leading order (NNLO) graphs have been enumerated by Kao et al. [28] within heavy-baryon chiral perturbation theory; however, no such program has been performed for the double radiative process. It is therefore possible that the remaining discrepancy between the measured branching ratio and the calculated branching ratio may reflect the neglect of either certain NLO graphs or all NNLO graphs in the existing calculations of the double radiative process. A modern calculation of double radiative capture within heavy baryon chiral perturbation theory would be welcome.

As mentioned in Sec. I, at appropriate kinematics the $\pi \pi \rightarrow \gamma \gamma$ annihilation graph in double radiative capture and $\pi \gamma \rightarrow \pi \gamma$ scattering graph in radiative pion photoproduction are potentially sensitive to the charged pion polarizability. Indeed, a recent experiment at MAMI [11], and an earlier ex- 
periment at Lebedev [44], have attempted to extract the polarizability from the $\pi \gamma \rightarrow \pi \gamma$ contribution to the $\gamma p \rightarrow \gamma \pi^{+} n$ reaction. Although, for threshold double radiative capture the pion polarizability effects are too small to be measurable, the dominant contribution of the $\pi \pi$ graph and vanishing contributions of the $\pi \mathrm{N}$ graphs do make the process an interesting yardstick for related theoretical studies of radiative pion production.

\section{Discussion of results for deuterium}

In comparing our results for hydrogen and deuterium we focus on the ratio $R(2 \gamma / 1 \gamma)$ and the two photon kinematical distributions. A quantitative comparison of absolute branching ratios is more difficult and less meaningful as pion absorption on nucleon pairs is important for deuterium but inapplicable for hydrogen.

Using our measured branching ratios for double radiative capture and the published branching ratios for single radiative capture we derived values for $R(2 \gamma / 1 \gamma)$ of $(7.68 \pm 0.69$ (stat.) \pm 0.79 (syst.) $) \times 10^{-5}$ for hydrogen and $\left(5.44 \pm_{0.46}^{0.34}\right.$ (stat.) \pm 0.42 (syst.) $) \times 10^{-5}$ for deuterium (Sec. VI A). The results imply a decrease in $R(2 \gamma / 1 \gamma)$ from hydrogen to deuterium by about $(29 \pm 12) \%$ (one small caveat is our detection of photon pairs is limited to $y>-0.1$ and $\omega>20 \mathrm{MeV})$. In addition, while the angular distributions are similar for hydrogen and deuterium, the energy distributions show evidence of lower average photon energies in deuterium (see Fig. 6). ${ }^{7}$

The decrease in $R(2 \gamma / 1 \gamma)$ and the change in photon energies from hydrogen to deuterium are probably not surprising. Single radiative capture and double radiative capture involve different reaction mechanisms and different momentum transfer, and therefore effects such as Pauli blocking, initial-state wavefunctions, final-state interactions, etc., may be different for these two processes. For example, the lower photon energies in deuterium capture compared to hydrogen capture may perhaps reflect the fact that energy is imparted to the spectator neutron through the final-state interactions in the deuterium process. In addition, the smaller $R(2 \gamma / 1 \gamma)$ ratio in deuterium capture compared to hydrogen capture may perhaps reflect a greater effect from Pauli blocking at the smaller momentum transfer of the double radiative process. Of course, to properly address the hydrogen-deuterium comparison a detailed calculation of deuterium capture is needed.

\footnotetext{
${ }^{7}$ Note that although the photon detection efficiencies were significantly different in hydrogen and deuterium their energy dependences were very similar (see Sec. VE) for details)
} 


\section{Comparison to nuclear double radiative capture.}

The world data for double radiative capture on complex nuclei consist of results for ${ }^{9} \mathrm{Be}$ and ${ }^{12} \mathrm{C}$ by Deutsch et al. [12] at CERN and results for ${ }^{12} \mathrm{C}$ from Mazzucato et al. [13] at TRIUMF. The experiment of Deutsch et al. obtained a partial branching ratio of $(1.0 \pm 0.1) \times 10^{-5}$ for ${ }^{9} \mathrm{Be}$ and $(1.4 \pm 0.2) \times 10^{-5}$ for ${ }^{12} \mathrm{C}$ for photon energies exceeding $25 \mathrm{MeV}$. The experiment of Mazzucato et al. obtained a partial branching ratio of $(1.2 \pm 0.2) \times 10^{-5}$ for ${ }^{12} \mathrm{C}$ for photon energies exceeding $17 \mathrm{MeV}$. Both experiments reported an increased yield of photon pairs at large opening angles although Mazzucato et al. have suggested that target contamination by hydrogenous materials may perhaps be responsible. The Mazzucato et al. experiment also reported a preference for unequal partition of photon energies.

In comparing the nuclear $(\pi, 2 \gamma)$ data to our $(\pi, 2 \gamma)$ data for hydrogen and deuterium we consider the ratio $R(1 \gamma / 2 \gamma)$ in order to avoid the complication of accounting for non-radiative processes in complex nuclei. For ${ }^{12} \mathrm{C}$, we derived values for $R(2 \gamma / 1 \gamma)$ of $(7.2 \pm 1.3) \times 10^{-4}$ from Mazzucato et al. and $(8.4 \pm 1.3) \times$ $10^{-4}$ from Deutsch et al. when normalized using the branching ratio for single radiative capture from Bistirlich et al. [45]. For ${ }^{9} \mathrm{Be}$, we obtained a value for $R(2 \gamma / 1 \gamma)$ of $(4.8 \pm 1.3) \times 10^{-4}$ when normalized using the mean branching ratio for single radiative capture from the neighboring ${ }^{7} \mathrm{Li}^{-10} \mathrm{~B}$ nuclei [46]. Clearly as summarized in Table VII - the values of $R(2 \gamma / 1 \gamma)$ on light nuclei are much larger than the corresponding ratios for hydrogen $(7.68 \pm 0.69 \pm 0.79) \times 10^{-5}$ and deuterium $\left(5.44 \pm_{0.46}^{0.34}\right.$ (stat.) \pm 0.42 (syst.) $\times 10^{-5}$. This increase in $R(2 \gamma / 1 \gamma)$ from $Z=1$ to light nuclei was anticipated by several authors $[5,26]$ as an evolution from predominantly annihilation dynamics in S-state capture to predominantly bremsstrahlung dynamics in atomic $\mathrm{P}$ states (see Sec. II C for details). For instance, an increase in $R(2 \gamma / 1 \gamma)$ from $\ell=0$ capture to $\ell=1$ capture of approximately ten-fold was predicted by Beder in Ref. [5].

Another difference between our data for $Z=1$ and earlier data for complex nuclei is the photon energy spectra. For $Z=1$ the energy spectra (Fig. 6 ) indicate a preference for the equal partition of the photon energies. For ${ }^{12} \mathrm{C}$ the energy spectra (Refs. [12] and [13]) indicate a preference for the unequal partition of the photon energies. This difference between $\ell=0$ capture and $\ell=1$ capture was also anticipated in early theoretical studies of double radiative capture by Beder [5] and Christillin and Ericson [26], and likewise reflects the evolving dynamics from annihilation in S-state capture to bremsstrahlung in P-state capture.

Another observation of experiments on nuclei was a backward peak in the angular distribution of the photon pairs. This feature was not predicted by the earlier calculation of Christillin and Ericson [26] but was obtained in the later calculation of Gil and Oset [27]. Unfortunately, the intense background of back-to-back photon pairs from $\pi^{o} \rightarrow \gamma \gamma$ decay made unthinkable the measurement of $Z=1$ double radiative capture at large opening angles.

In summary, we claim the large difference in $R(2 \gamma / 1 \gamma)$ between the ele- 
mentary and nuclear processes, and significant difference in the energy partition between the elementary and nuclear processes, are clear evidence of the evolving dynamics of the double radiative process from S-state to P-state capture. This conclusion implies that earlier arguments for the sensitivity of the $\left(\pi^{-}, 2 \gamma\right)$ nuclear reaction to the pion field in the nuclear medium, that were founded on the dominance of the $\pi \pi \rightarrow \gamma \gamma$ mechanism, are likely not justified.

\section{CONCLUSION}

We have performed the first measurements of double radiative capture on pionic hydrogen and pionic deuterium. The measurements were performed using the RMC spectrometer at the TRIUMF cyclotron by recording photon pairs from pion stops in liquid hydrogen and deuterium targets. We have determined the absolute branching ratios for double radiative capture, the ratios $R(2 \gamma / 1 \gamma)$ of double radiative capture to single radiative capture, and the energy-angle distributions of the resulting photon pairs.

For hydrogen we obtained an absolute branching ratio of (3.02 \pm 0.27 (stat.) \pm 0.31 (syst. $)) \times 10^{-5}$ and ratio $R(2 \gamma / 1 \gamma)$ of $(7.68 \pm 0.69($ stat $) \pm$ $0.79($ syst $)) \times 10^{-5}$. The measured branching ratios and energy-angle distributions are in fair agreement with the theoretical predictions of Joseph [25], Lapidus and Musakanov [4] and Beder [5] and lend support to the theoretical claim of a $\pi \pi \rightarrow \gamma \gamma$ annihilation mechanism. This mechanism implies that double radiative capture from $\ell=0$ orbitals inherits an intriguing sensitivity to the nucleon's pion cloud and the $\pi \pi \rightarrow \gamma \gamma$ vertex.

For hydrogen the measured and calculated branching ratio do, however, differ by approximately $40 \%$. One possible explanation is the neglect of either certain NLO graphs or all NNLO graphs in the existing calculations of the double radiative process. Given the relationship between double radiative pion capture $\pi^{-} p \rightarrow \gamma \gamma n$ and radiative pion photoproduction $\gamma p \rightarrow \gamma \pi^{+} n$ - and their relevance to the determination of the pion's polarizability - we believe that resolving this discrepancy is of some importance. A modern calculation of double radiative pion capture within heavy-baryon chiral perturbation theory would be welcome.

For deuterium we obtained an absolute branching ratio of $\left(1.42 \pm_{0.12}^{0.09}\right.$ (stat.) \pm 0.11 (syst.) $) \times 10^{-5}$ and ratio $R(2 \gamma / 1 \gamma)$ of $\left(5.44 \pm_{0.46}^{0.34}\right.$ (stat) \pm $0.42($ syst $)) \times 10^{-5}$. We found that the deuterium ratio $R(2 \gamma / 1 \gamma)$ is smaller than the hydrogen ratio $R(2 \gamma / 1 \gamma)$ by $(29 \pm 12) \%$. We also found the photon energies are shifted somewhat to lower energies in the deuterium data compared to the hydrogen data. The decrease in $R(2 \gamma / 1 \gamma)$ and shift in photon energies are possibly revealing the spectator neutron's role via final-state interactions and Pauli blocking effects.

Lastly, we compared our results for hydrogen and deuterium with the earlier results for beryllium and carbon. The relative branching ratios $R(2 \gamma / 1 \gamma)$ for light nuclei are nearly ten times larger than the corresponding values for 
the hydrogen isotopes. In addition, the energy distributions are markedly different for the light nuclei and the hydrogen isotopes; the former favoring unequal energy partition with the latter favoring equal energy partition. These observations are consistent with the dynamics evolving from a $\pi \pi \rightarrow \gamma \gamma$ annihilation mechanism for $\ell=0$ capture to a $\pi \mathrm{N}$ bremsstrahlung mechanism for $\ell=1$ capture. This implies that nuclear double radiative pion capture may not inherit the high sensitivity to the pion field in the nuclear medium as was originally proposed.

\section{ACKNOWLEDGEMENTS}

We would like to thank the staff of the TRIUMF laboratory for the operation of the cyclotron and the maintenance of the RMC spectrometer. We would also like to thank Dr. Renée Poutissou for assistance with the data acquisition and Dr. Dennis Healey for assistance with the cyrogenic targets. We also thank Drs. Fearing, Kao, Oset and Wilkin for valuable communications on the theoretical aspects of the double radiative process. Lastly, we

are indebted the National Science Foundation (USA), Natural Sciences and Engineering Research Council (Canada), and Jeffress Memorial Trust and the William \& Mary Endowment for their financial support. 


\section{REFERENCES}

$\dagger$ Present address: SLAC, P.O. Box 20450, Stanford, CA 94309.

$\ddagger$ Present address: American Physical Society, One Physics Ellipse, College Park, MD, 20740.

[1] Norman M. Kroll and Malvin A. Ruderman, Phys. Rev. 93, 233 (1954).

[2] V. Bernard, N. Kaiser and Ulf-G. Meiner, Phys. Lett. B383, 116 (1996)/

[3] Harold W. Fearing, Thomas R. Hemmert, Randy Lewis and Christine Unkmeir, Phys. Rev. C 62, 054006 (2000).

[4] L. Lapidus and M. Musakhanov, Sov. Jour of Nucl. Phys., 15, 558 (1972).

[5] D. Beder, Nucl. Phys. B156, 482 (1979).

[6] D. Beder, Phys. Rev. C, 26, 555 (1982).

[7] J.B. Cammarata, Phys. Rev. C, 20, 705 (1979).

[8] T.E.O. Ericson and C. Wilkin, Phys. Lett. 57B, 345 (1975).

[9] Ebbe M. Nyman and Mannque Rho, Nucl. Phys. A287, 390 (1977).

[10] Saul Barshay, Phys. Lett. 76B, 384 (1978).

[11] J. Ahrens et al., Eur. Phys. J. A 23, 113 (2005)

[12] J. Deutsch, D. Favart, M. Lebrun, P. Lipnik, P. Macq and R. Prieels, Phys. Lett. 80B, 347 (1979).

[13] M. Mazzucato, B. Bassaleck, M.D. Hasinoff, T. Marks, J.M. Poutissou and M. Salomon, Phys. Lett. 96B, 43 (1980).

[14] I.M. Vasilevsky, V.V. Vishnyakov, A.F. Dunaytsev, Yu.D. Prokoshkin, V.I. Rykalin, and A.A. Tyapkin, Nucl. Phys. B9(1969)673.

[15] S. Tripathi et al., Phys. Rev. Lett. 89, 252501 (2002).

[16] P. A. Zolnierczuk, D.S. Armstrong, E. Christy, J.H. Clark, T. P. Gorringe, M. D. Hasinoff, M. A. Kovash, S. Tripathi and D. H. Wright, Phys. Lett. B 597, 131 (2004).

[17] P. A. Zolnierczuk, T. P. Gorringe, M. D. Hasinoff, M. A. Kovash, S. Tripathi and D. H. Wright, Phys. Lett. B 549, 301 (2002).

[18] C.J. Batty, Sov. J. Part. Nucl. 13, 71 (1982).

[19] T.B. Day, G.A. Snow and J. Sucher Phys. Rev. 118, 864 (1960).

[20] M. Leon and H.A. Bethe, Phys. Rev. 127, 636 (1962).

[21] E. Borie and M. Leon, Phys. Rev. A 21, 1460 (1980).

[22] T.P. Terada and R.S. Hayano, Phys. Rev. C 55, 73 (1997).

[23] K. Brueckner, R. Serber and K. Watson, Phys. Rev. 81, 575 (1951).

[24] W.W. Sapp, Jr., M. Eckhause, G.H. Miller, and R.E. Welsh, Phys. Rev. C 5, 690 (1972).

[25] D. W. Joseph, Nuovo Cimento XVI(1960)995.

[26] P. Christillin and T.E.O. Ericson, Phys. Lett. 87B, 163 (1979).

[27] A. Gil and E. Oset, Phys. Lett. 346B, 1 (1995).

[28] C. w. Kao, B. E. Norum and K. Wang, arXiv:hep-ph/0409081.

[29] J. Spuller, D. Berghofer, M.D. Hasinoff, R. Macdonald, D.F. Measday, M. Salomon, T. Suzuki, J.M. Poutissou, R. Poutissou, and J.K.P. Lee, Phys. Lett. 67B, 4 (1977).

[30] N.P. Samios, Phys. Rev. 121, 275 (1961).

[31] H. Fonvieille, N. Bensayah, J. Berthot, P. Bertin, M. Crouau, B. Bihoreau, G. Fournier, J. Miller, B. Saghai, C. Samour, P. Vernin, M. Giffon and R. Nahabetian, Phys. Lett. 233B, 60 (1989).

[32] V. L. Highland, M. Salomon, M. D. Hasinoff, E. Mazzucato, D. F. Measday, J. M. Poutissou and T. Suzuki, Nucl. Phys. A 365, 333 (1981). 
[33] R. MacDonald et al., Phys. Rev. Lett. 38, 746 (1977).

[34] D. H. Wright et al., Phys. Rev. C 57, 373 (1998).

[35] D.H. Wright et al., Nucl. Instrum. Methods A320, 249 (1992).

[36] N. M. M. Al-Qazzaz et al., Nucl. Instrum. Meth. 174, 35 (1980).

[37] J. B. Lindsay, C. Millerin, J. C. Tarle, H. Verweij and H. Wendler, Nucl. Instrum. Meth. 156, 329 (1978).

[38] H. Brafman, T. Glanzman, A.J. Lankford, J. Olsen and L. Paffrath, IEEE Trans. Nucl. Sci. NS-32, 336 (1985).

[39] P. Bennett et al., IEEE Trans. Nucl. Sci. NS-37, 1200 (1990).

[40] P. Bennett, TRIUMF Internal Technical Note (1991).

[41] R. Brun, F. Bruyant, M. Maire, A.C. McPherson and P. Zanarini, GEANT3(1986); CERN report no. DD/EE/84-1 (unpublished).

[42] H.C. Schroder et al., Phys. Lett. B469, 25 (1999).

[43] P. Hauser et al., Phys. Rev. C 58 R1869 (1998).

[44] T.A. Aibergenov, P.S. Baranov, O.D. Beznisko, S.N. Cherepniya, L.V. Filkov, A.A. Nafikov, A.I.'Osadchii, V.G. Raevskii, L.N. Shtarkov and E.I. Tamm, Czech. J. Phys. B36 948 (1986).

[45] J. A. Bistirlich, K. M. Crowe, A. S. L. Parsons, P. Skarek and P. Truoel, Phys. Rev. C 5, 1867 (1972).

[46] H. W. Baer, K. M. Crowe, and P. Truol, in "Advances in Nuclear Physics" (Plenum, New York, Vol. 77), Vol. 9, Chap. 3.. 


\section{TABLES}

TABLE I. Compilation of previously published branching ratios for negative pion capture on hydrogen and deuterium.

\begin{tabular}{lcc}
\hline \hline & & \\
capture mode & expt. & branching ratio \\
& & \\
\hline & Spuller et al. $[29]$ & $0.607 \pm 0.004$ \\
$\pi^{-} p \rightarrow \pi^{o} n$ & Spuller et al. $[29]$ & $0.393 \pm 0.003$ \\
$\pi^{-} p \rightarrow \gamma n$ & Samios [30] & $(2.72 \pm 0.19) \times 10^{-3}$ \\
$\pi^{-} p \rightarrow e^{+} e^{-} n$ & Highland et al. $[32]$ & $0.739 \pm 0.010$ \\
$\pi^{-} d \rightarrow n n$ & Highland et al. $[32]$ & $0.261 \pm 0.004$ \\
$\pi^{-} d \rightarrow \gamma n n$ & MacDonald et al. $[33]$ & $(1.45 \pm 0.19) \times 10^{-4}$ \\
$\pi^{-} d \rightarrow \pi^{o} n n$ & & \\
& & \\
\hline \hline
\end{tabular}


TABLE II. Summary of tracking cuts for positrons and electrons and "nonwrap arounds" and "wrap arounds". The upper rows are the hydrogen values and the lower rows are the deuterium values.

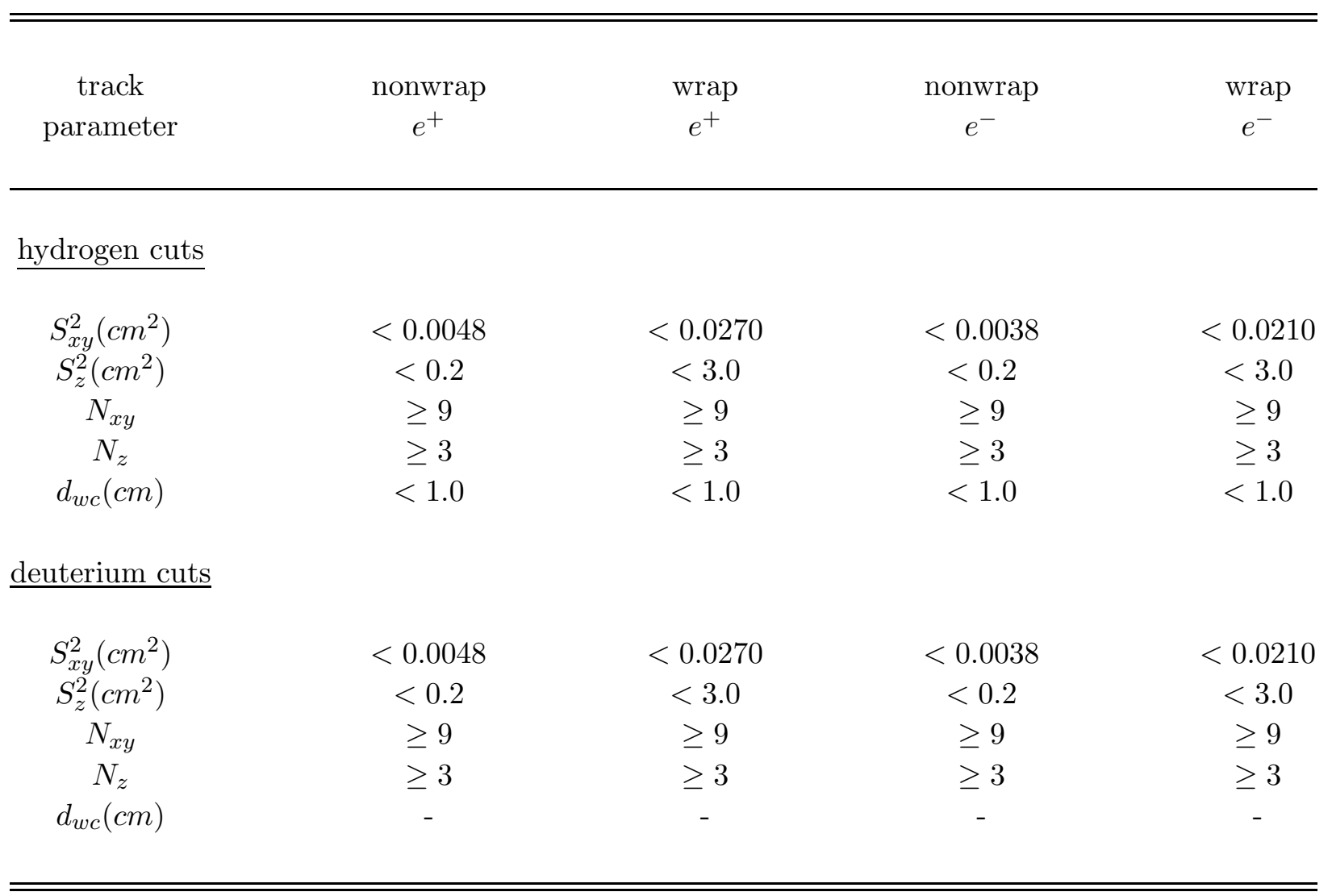


TABLE III. Summary of the photon cuts for "non-wrap around" photons (i.e. containing no wrap-around tracks) and "wrap around" photons (i.e. containing wrap-around tracks). The upper rows are the hydrogen values and the lower rows are the deuterium values.

\begin{tabular}{ccc}
\hline \hline & & \\
photon & nonwrap & wrap \\
parameter & & \\
& & \\
\hline & & \\
hydrogen cuts & & $<7.0$ \\
\hline & $<6.0$ & $<7.0$ \\
$d_{x y}(\mathrm{~cm})$ & $<6.0$ & $<8$ \\
$d_{z}(\mathrm{~cm})$ & $<8$ & $-12<z<+14$ \\
$r_{\text {close }}(\mathrm{cm})$ & $-12<z<+14$ & \\
$z_{\text {close }}(\mathrm{cm})$ & & $<7.0$ \\
deuterium cuts & $<6.0$ & $<14.0$ \\
$d_{x y}(\mathrm{~cm})$ & $<12.0$ & $<8$ \\
$d_{z}(\mathrm{~cm})$ & $<8$ & $-25<z<+27$ \\
$r_{\text {close }}(\mathrm{cm})$ & $-25<z<+27$ & \\
$z_{\text {close }}(\mathrm{cm})$ & $<$ & \\
\hline \hline
\end{tabular}


TABLE IV. Summary of background estimates and background limits for the hydrogen analysis and the deuterium analysis. The first row is the two-photon events with summed energies 80-150 MeV before the background subtraction and the last low is two-photon events with summed energies 80-150 MeV after the background subtraction.

\begin{tabular}{ccc}
\hline \hline & & \\
background source & $\mathrm{H}_{2}$ & $\mathrm{D}_{2}$ \\
\hline & & \\
\hline 80-150 MeV events before sub. & 566 & 327 \\
& & $2 \pm 1$ \\
$\pi^{o}$ bkd. & $53 \pm 30$ & $20 \pm 2$ \\
$\pi \pi$ bkd. & $40 \pm 8$ & $15 \pm 4$ \\
$\mu \pi$ bkd. & 0 & $<1$ \\
Dalitz bkd. & $<2$ & $<20$ \\
nucl. $(\pi, 2 \gamma)$ & $<10$ & $292 \pm \frac{19}{27}$ \\
80-150 MeV events after sub. & $465 \pm 40$ & \\
\hline \hline
\end{tabular}

TABLE V. Summary of quantities for the calculations of the total branching ratios of double radiative capture on pionic hydrogen and pionic deuterium. Note that the quoted acceptances assumes the energy-angle distributions of Beder.

\begin{tabular}{ccc}
\hline \hline & & \\
quantity & $\mathrm{H}_{2}$ & $\mathrm{D}_{2}$ \\
& & \\
\hline & & \\
$\mathrm{N}_{\gamma \gamma}$ & $465 \pm_{40}^{39}$ & $292 \pm \pm_{27}^{19}$ \\
$\mathrm{~N}_{\pi^{-}}$ & $(3.10 \pm 0.03) \times 10^{11}$ & $(3.07 \pm 0.15) \times 10^{11}$ \\
$\epsilon \Delta \Omega$ & $0.66 \times 10^{-4}$ & $0.90 \times 10^{-4}$ \\
$\mathrm{~F}$ & $0.90 \pm 0.09$ & $0.96 \pm 0.06$ \\
$\mathrm{C}_{\text {stop }}$ & $0.85 \pm 0.01$ & $0.85 \pm 0.01$ \\
$\mathrm{C}_{b m}$ & 0.98 & 0.91 \\
absolute B.R. & $3.02 \pm 0.27($ stat $) \pm 0.31$ (syst $) \times 10^{-5}$ & $1.42 \pm_{0.12}^{0.09}($ stat $) \pm 0.11(\mathrm{syst}) \times 10^{-5}$ \\
ratio $\mathrm{R}(2 \gamma / 1 \gamma)$ & $7.68 \pm 0.69($ stat $) \pm 0.79($ syst $) \times 10^{-5}$ & $5.44 \pm_{0.46}^{0.34}($ stat $) \pm 0.42($ syst $) \times 10^{-5}$ \\
& & \\
\hline \hline
\end{tabular}


TABLE VI. Sensitivity of the absolute branching ratio to the analysis parameters, different data-sets and the theoretical energy-angle distributions for the hydrogen analysis and the deuterium analysis. We have combined in quadrature the various statistical and systematic uncertainties.

\begin{tabular}{ccr}
\hline \hline & & \\
cuts/data-set/theory & $\mathrm{H}_{2}$ & $\mathrm{D}_{2}$ \\
& & \\
\hline & & \\
$80<\omega_{1}+\omega_{2}<150, y>0.0$ & $(2.94 \pm 0.41) \times 10^{-5}$ & $\left(1.39 \pm_{0.16}^{0.14}\right) \times 10^{-5}$ \\
$80<\omega_{1}+\omega_{2}<150, y>-0.1$ & $(3.02 \pm 0.41) \times 10^{-5}$ & $\left(1.42 \pm_{0.16}^{0.14}\right) \times 10^{-5}$ \\
$80<\omega_{1}+\omega_{2}<150, y>-0.2$ & $(3.14 \pm 0.41) \times 10^{-5}$ & $\left(1.39 \pm_{0.16}^{0.14}\right) \times 10^{-5}$ \\
& & $\left(1.48 \pm_{0.17}^{0.14}\right) \times 10^{-5}$ \\
$70<\omega_{1}+\omega_{2}<150, y>-0.1$ & $(3.11 \pm 0.41) \times 10^{-5}$ & $\left(1.48 \pm_{0.17}^{0.14}\right) \times 10^{-5}$ \\
$60<\omega_{1}+\omega_{2}<150, y>-0.1$ & $(3.17 \pm 0.41) \times 10^{-5}$ & $\left(1.42 \pm_{0.16}^{0.14}\right) \times 10^{-5}$ \\
& & $\left(1.38 \pm_{0.16}^{0.14}\right) \times 10^{-5}$ \\
$80<\omega_{1}+\omega_{2}<160, y>-0.1$ & $(3.11 \pm 0.41) \times 10^{-5}$ & $\mathrm{n} / \mathrm{a}$ \\
$80<\omega_{1}+\omega_{2}<170, y>-0.1$ & $(3.05 \pm 0.41) \times 10^{-5}$ & $\mathrm{n} / \mathrm{a}$ \\
& & \\
hydrogen data-set I & $(2.94 \pm 0.49) \times 10^{-5}$ & $\left(1.42 \pm_{0.16}^{0.14}\right) \times 10^{-5}$ \\
hydrogen data-set II & $(3.15 \pm 0.49) \times 10^{-5}$ & $\left(1.22 \pm_{0.14}^{0.12}\right) \times 10^{-5}$ \\
Beder, all graphs & & $\mathrm{n} / \mathrm{a}$ \\
Beder, $\pi \pi$ graphs & $(3.02 \pm 0.41) \times 10^{-5}$ & \\
phase space & $(2.63 \pm 0.41) \times 10^{-5}$ & $(3.16 \pm 0.41) \times 10^{-5}$ \\
& & \\
\hline \hline
\end{tabular}

TABLE VII. Summary of determinations of ratios $R(2 \gamma / 1 \gamma)$ from our hydrogen and deuterium data and earlier beryllium and carbon data. Note that for beryllium and carbon the ratios are for photon energies $>25 \mathrm{MeV}$ in Deutsch et al. and $>7 \mathrm{MeV}$ Mazzucato et al.

\begin{tabular}{lcc}
\hline \hline & \multicolumn{1}{c}{ Ref. } & $R(2 \gamma / 1 \gamma)$ \\
target & this work & $(7.68 \pm 0.69 \pm 0.79) \times 10^{-5}$ \\
& this work & $\left(5.44 \pm \pm \pm_{0.46}^{0.34}(\right.$ stat. $) \pm 0.42($ syst. $) \times 10^{-5}$ \\
& Deutsch et al. $[12]$ & $(4.8 \pm 1.3) \times 10^{-4}$ \\
${ }^{1} \mathrm{H}$ & Deutsch et al. $[12]$ & $(7.4 \pm 1.3) \times 10^{-4}$ \\
${ }^{2} \mathrm{H}$ & Mazzucato et al. $[13]$ & \\
${ }^{9} \mathrm{Be}$ & & \\
${ }^{12} \mathrm{C}$ & & \\
${ }^{12} \mathrm{C}$ & & \\
&
\end{tabular}




\section{FIGURES}

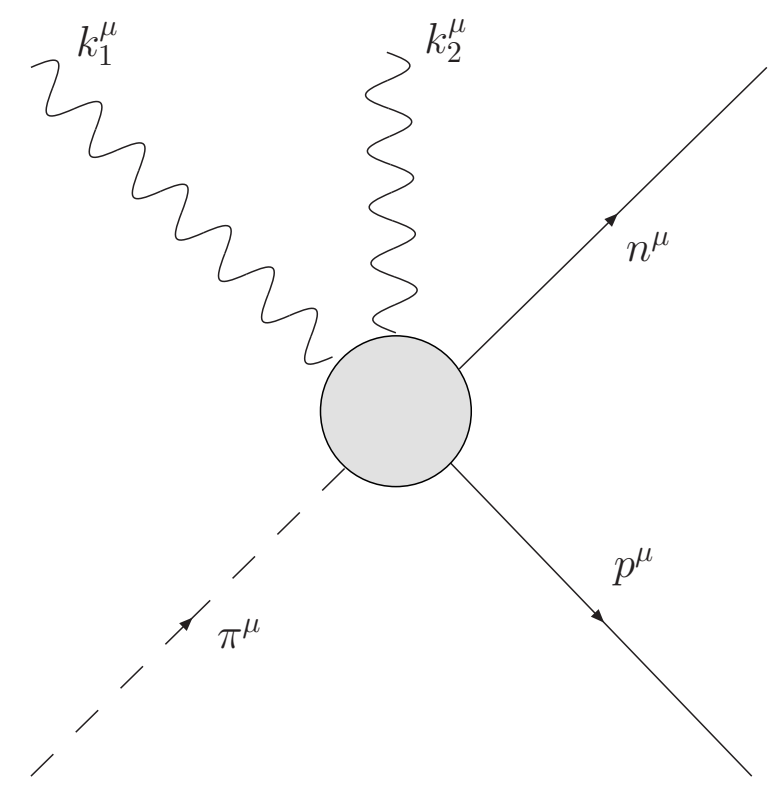

FIG. 1. Definitions of kinematical quantities in the $\pi^{-} p \rightarrow \gamma \gamma n$ elementary process.

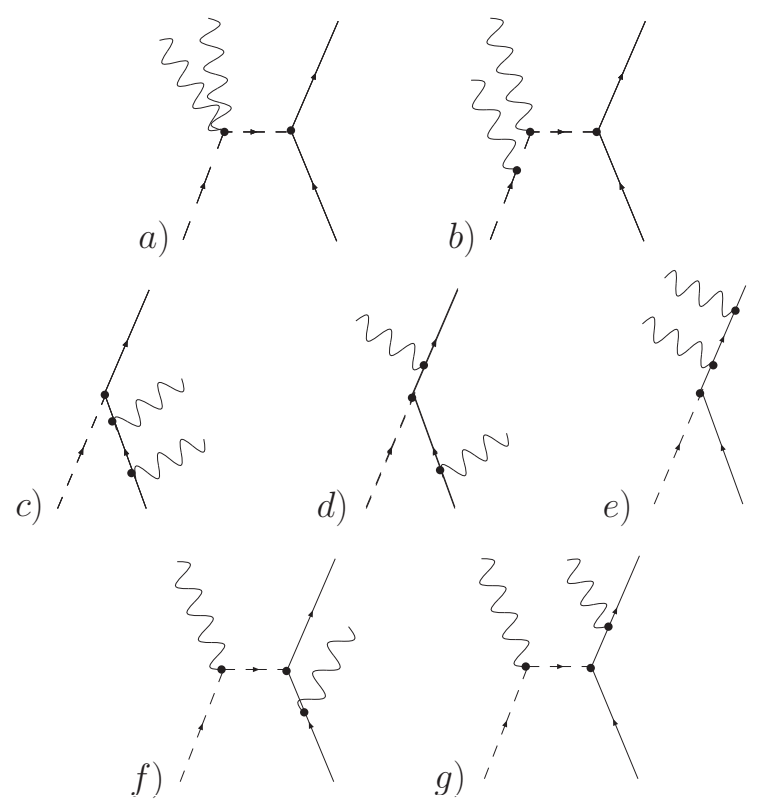

FIG. 2. Tree-level contributions to double pion radiative capture in the $\pi N \gamma$ effective Lagrangian approach of Beder. Diagrams a-b) are denoted the $\pi \pi$ graphs, c-e) the NN graphs, and f-g) $\pi \mathrm{N}$ graphs. 

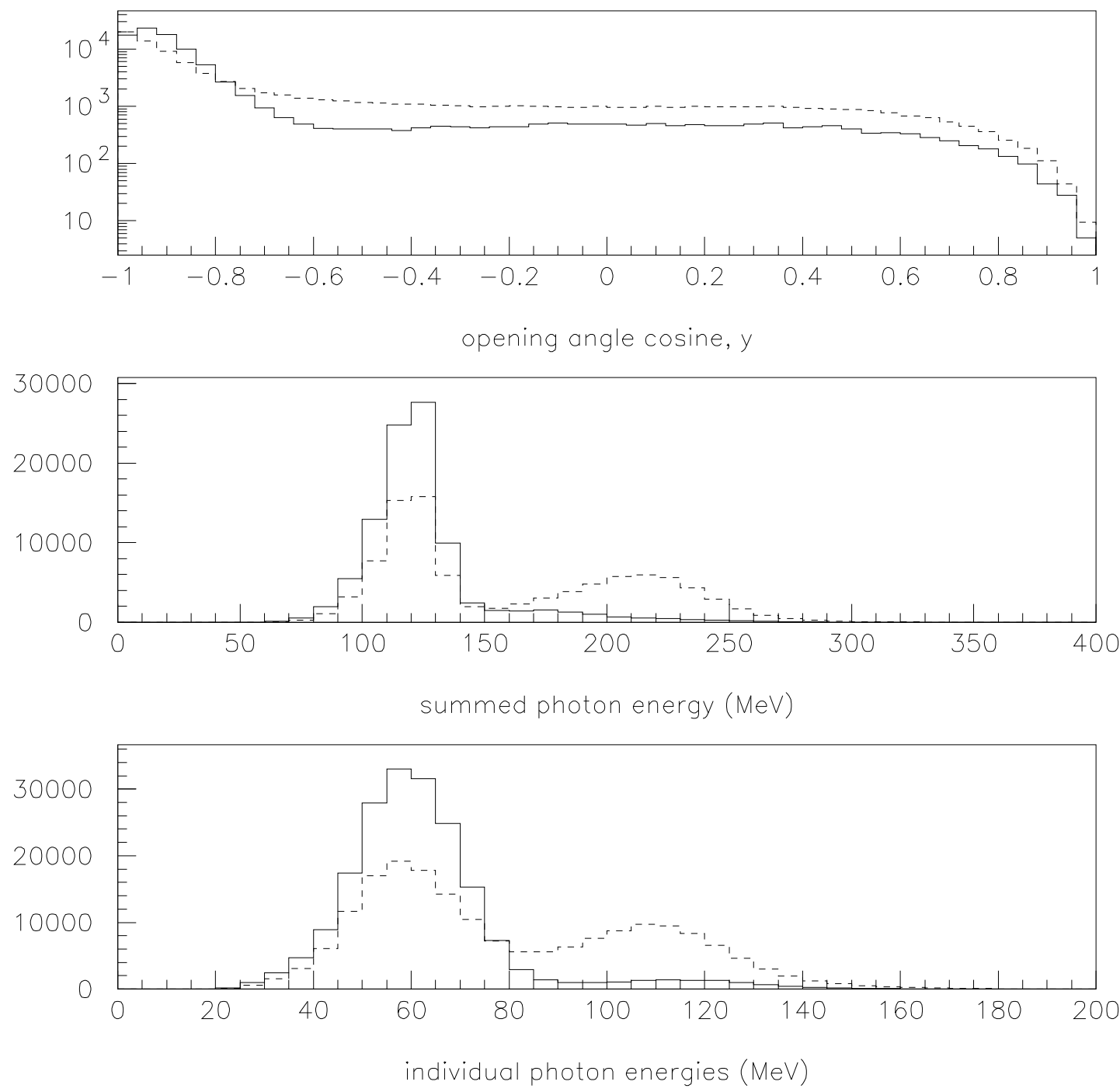

FIG. 3. The opening angle (top), summed energy (center) and individual energy (bottom) spectra of the two-photon events passing the tracking cuts and photon cuts in the hydrogen data-set (solid histogram) and the deuterium data-set (dashed histogram). The $\pi^{o}$ background is apparent as the events with $y<-0.7$ in the opening angle spectrum and the $\pi \pi$ background is apparent as the events with $\omega_{1}+\omega_{2}>150 \mathrm{MeV}$ in the sum energy spectrum. To facilitate their comparison the hydrogen spectra and deuterium spectra are normalized to equal numbers of photon-pair events. 

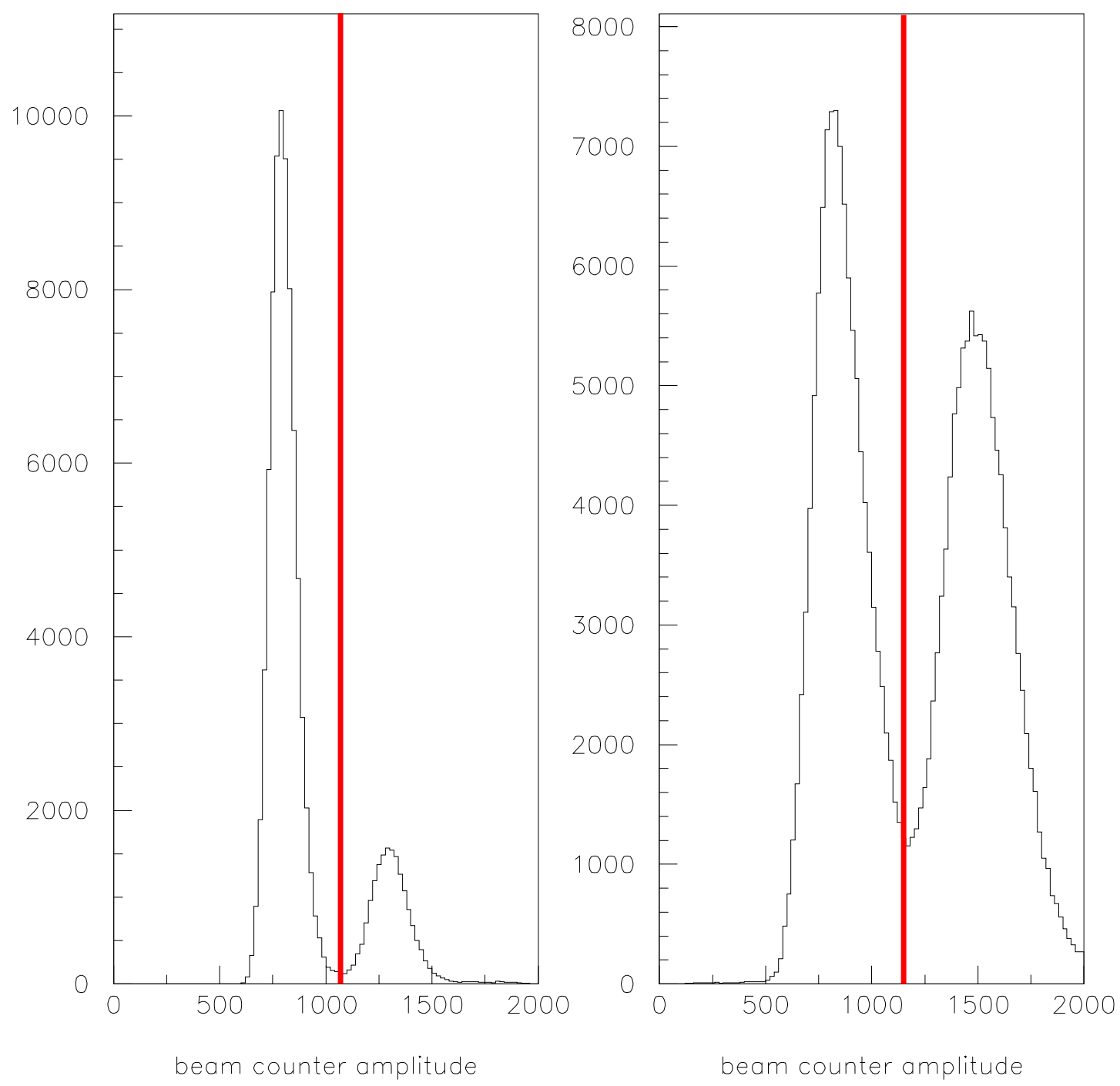

FIG. 4. The beam amplitude spectra for the hydrogen data-set photon pairs (lefthand plot) and deuterium data-set photon pairs (righthand plot). The plots show the single pion stops associated with real photon pairs (the lower amplitude peak) and two pion stops associated with accidental photon pairs (the higher amplitude peak). The beam counter cuts, at Chan. 1069. in the hydrogen data-set and Chan. 1150 in the deuterium data-set, are indicated by vertical bars. 


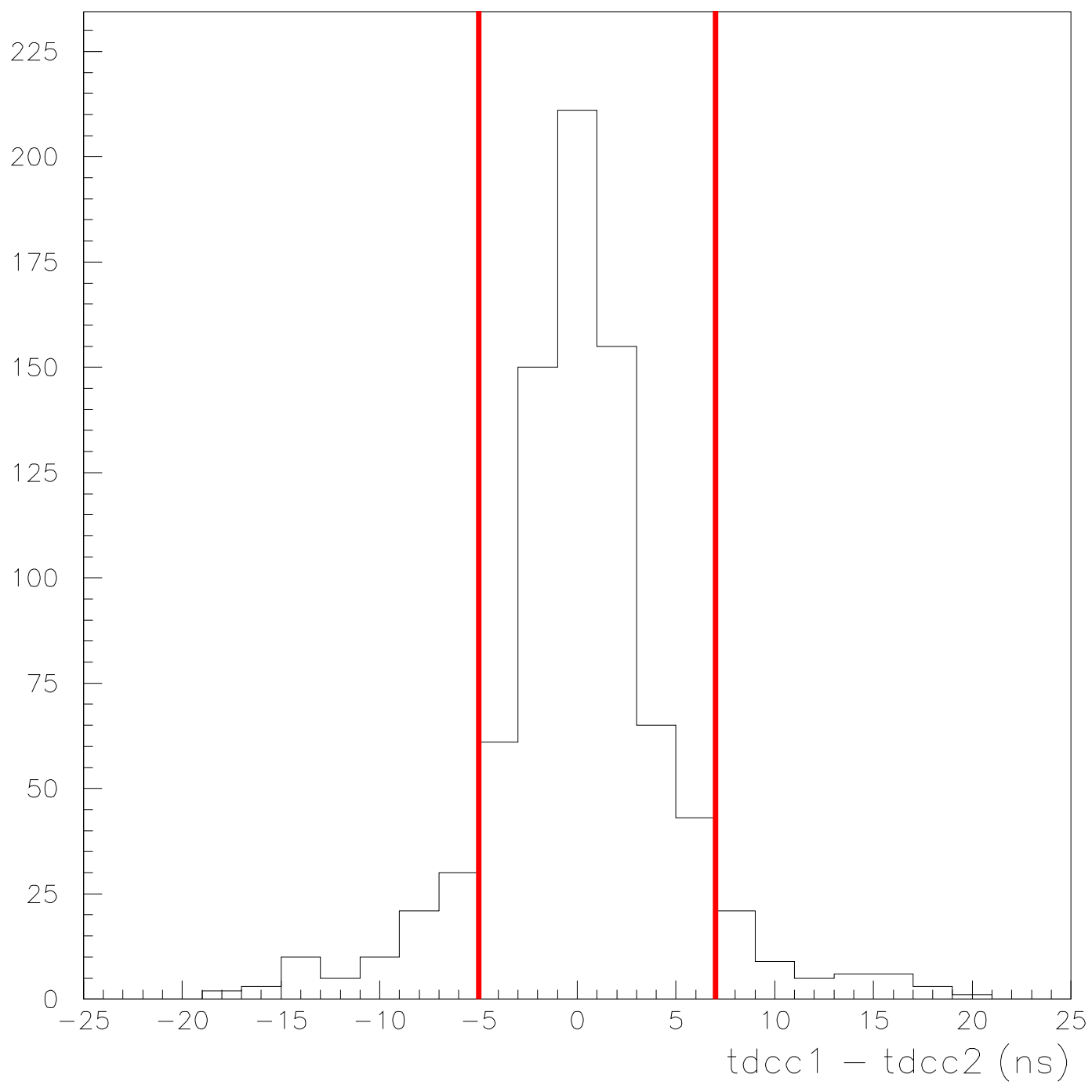

FIG. 5. The time difference (ns) between two C-counters hits for photon pairs in deuterium that pass all cuts with the exception of the C-counter timing cut. The events outside the coincidence window $-5<\left(t_{C 1}-t_{C 2}\right)<+7$ ns are dominated by $\mu \pi$ and $\pi \pi$ baclground. 

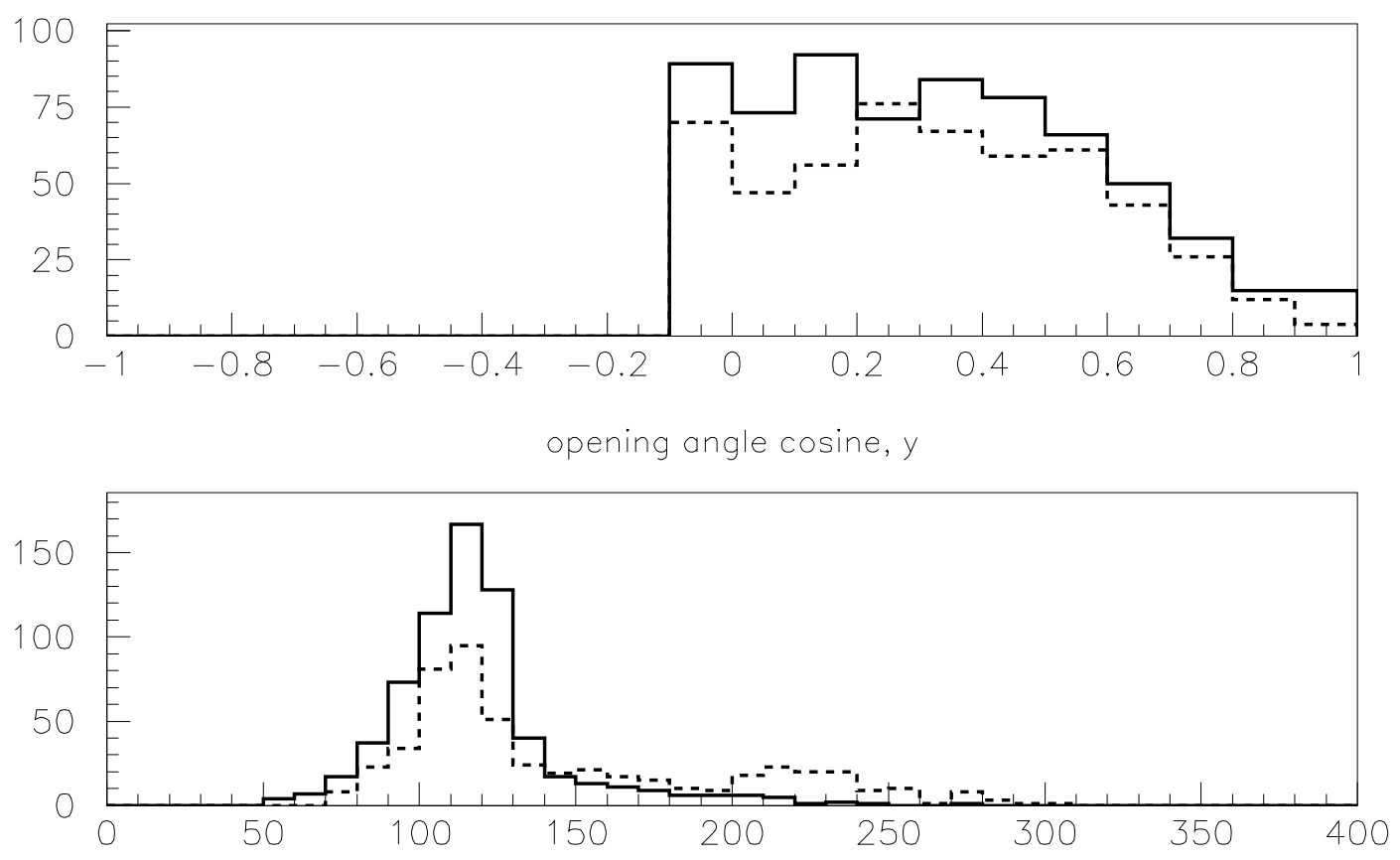

summed photon energy (MeV)

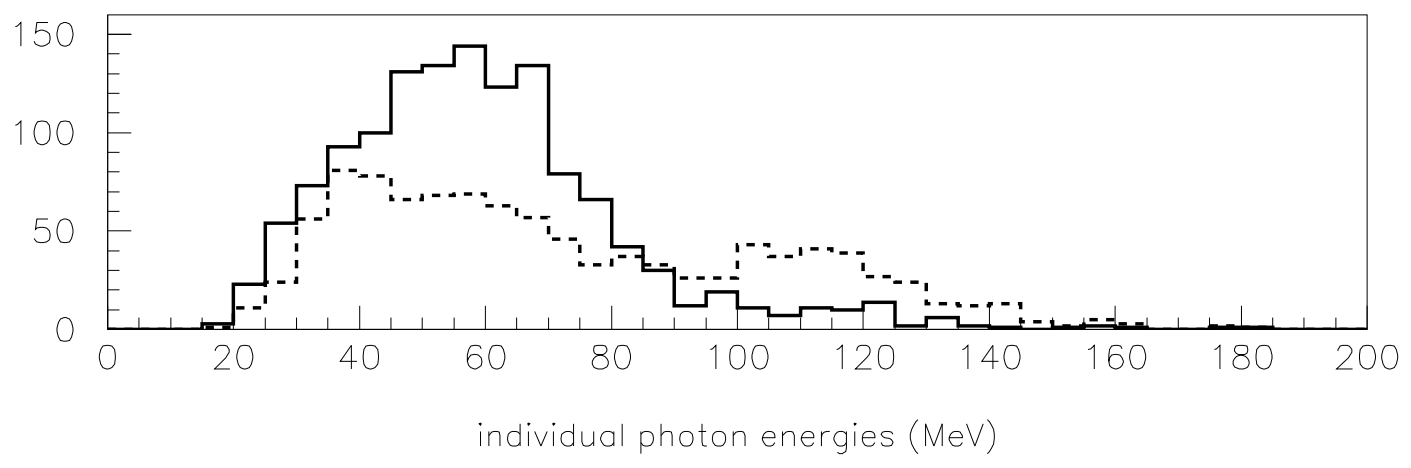

FIG. 6. The opening angle (top), summed energy (center) and individual energy (bottom) spectra for photon pairs after all cuts for the hydrogen data-set (solid histogram) and deuterium data-set (dashed histogram). The hydrogen spectra contain a total of 665 photon-pair events with $y>-0.1$ The deuterium spectra contain a total of 521 photon-pair events with $y>-0.1$ 


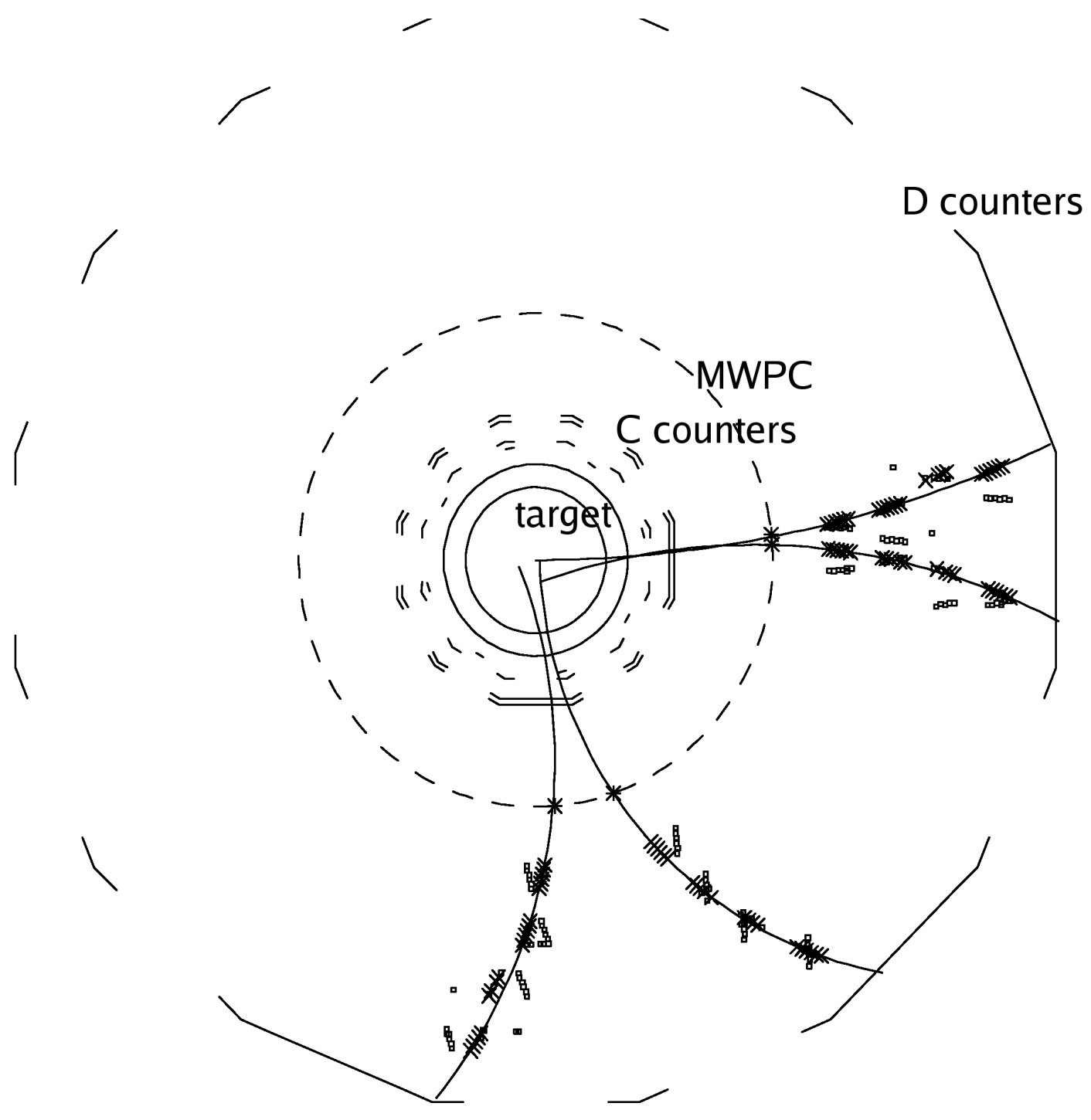

RUN

FIG. 7. A typical double radiative capture event showing the trajectories of the two $e^{+} e^{-}$pairs, the circle fits to the four $e^{+} e^{-}$tracks, and the fired $\mathrm{C}$ and $\mathrm{D}$ counters. 


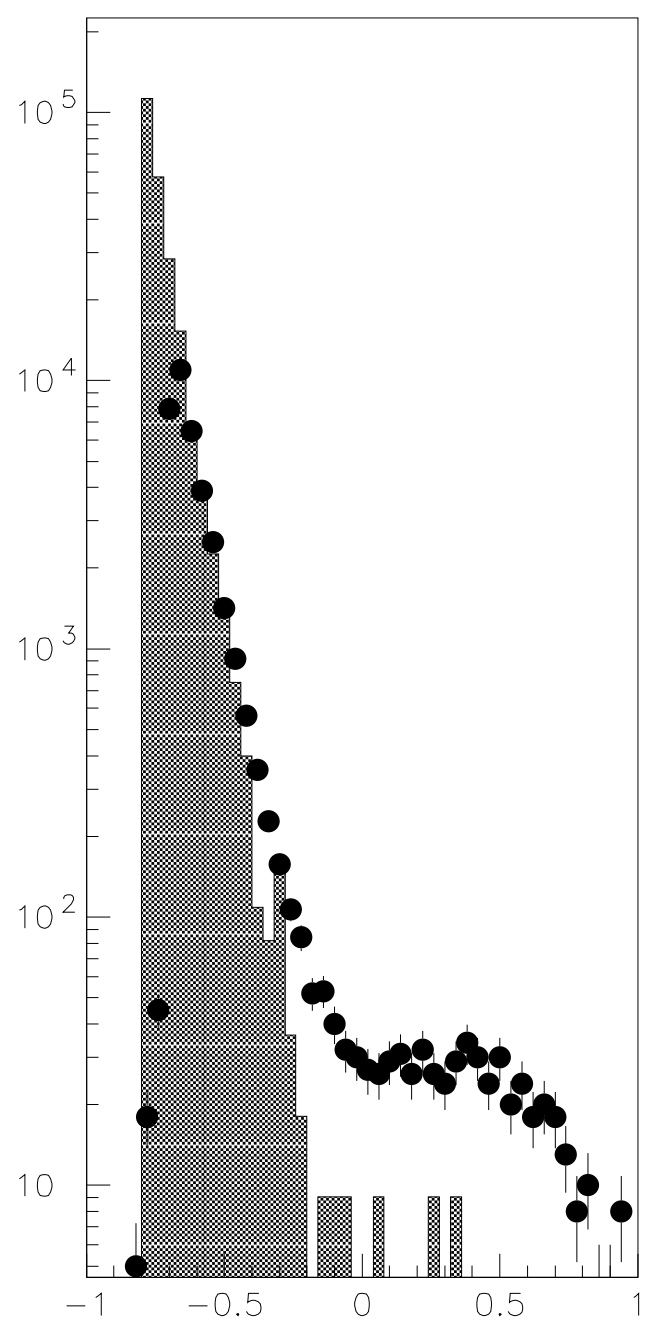

opening angle cosine, y

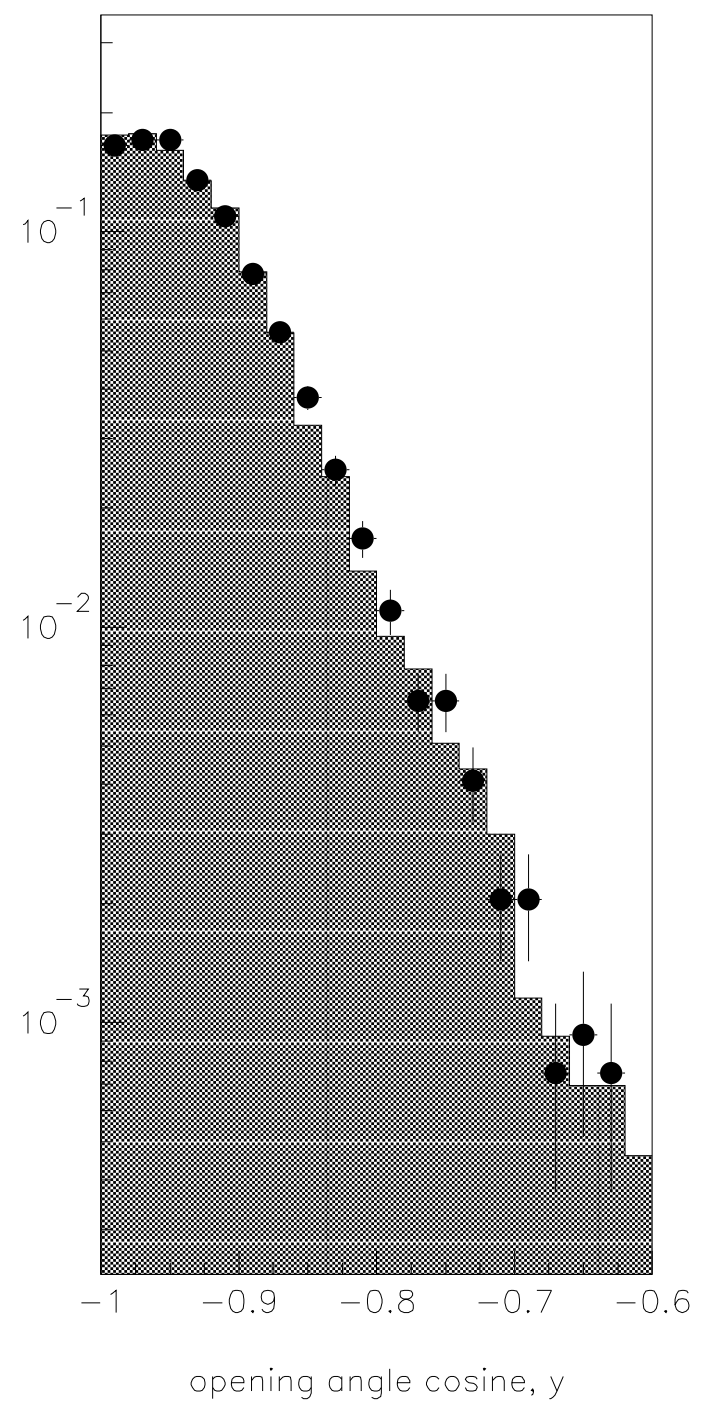

FIG. 8. Comparison of Monte Carlo and experimental data for photon-pairs from $\pi^{o}$ decay for the $\gamma \gamma$ trigger and $-0.8<y+1.0$ (lefthand plot) and the $\pi^{o}$ trigger and $-1.0<y-0.6$ (righthand plot). The solid histograms are the Monte Carlo results and the data points are the experimental results. (The apparent difference between Monte Carlo and experimental data for $y<-0.75$ in the $\gamma \gamma$ trigger data is a consequence of a loose y-cut that was applied in the initial processing of the experimental data ) 


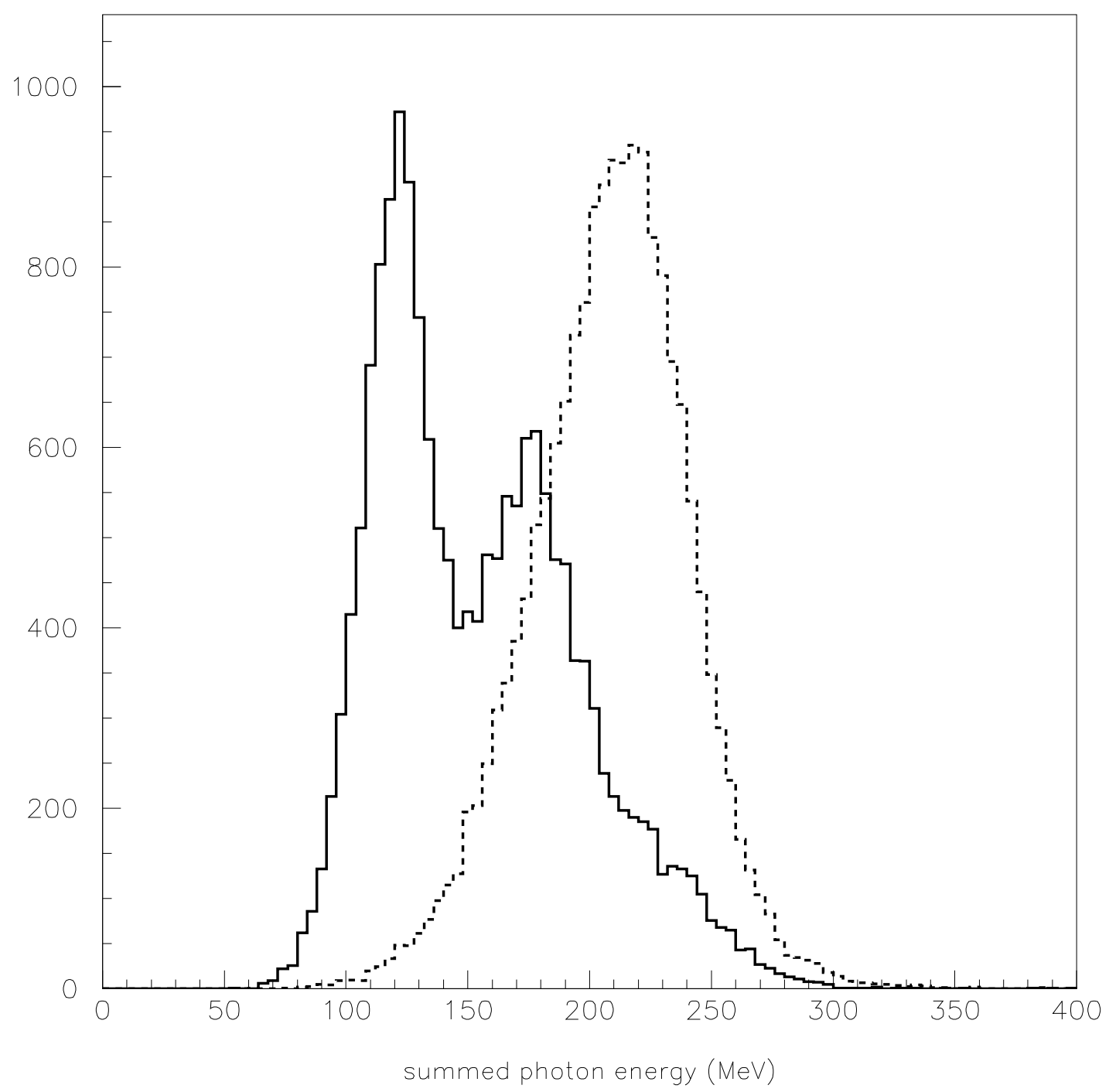

FIG. 9. The photon sum energy spectrum for hydrogen (solid curve) and deuterium (dashed curve) for events that fail the beam amplitude cut, i.e. accidental $\gamma \gamma$ coincidences due to multiple pions in a single beam pulse. In deuterium the accidental coincidences are dominated by single radiative capture. In hydrogen the accidental coincidences originate from pion charge exchange and single radiative capture. 


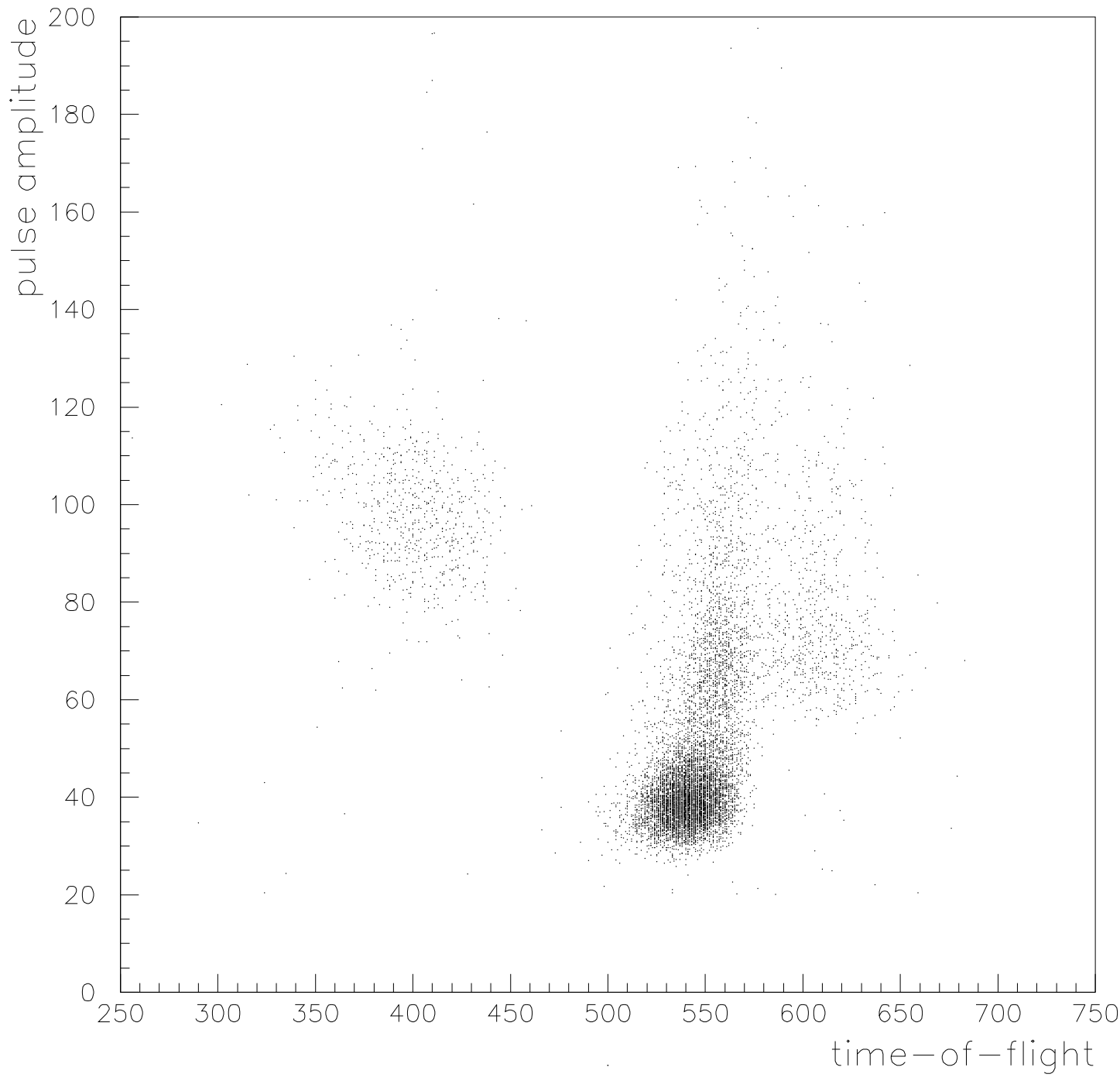

FIG. 10. Beam counter amplitude versus time-of-flight for incoming beam particles in the deuterium experiment. The pions are top-left, electrons are bottom-center and muons are right-center. 

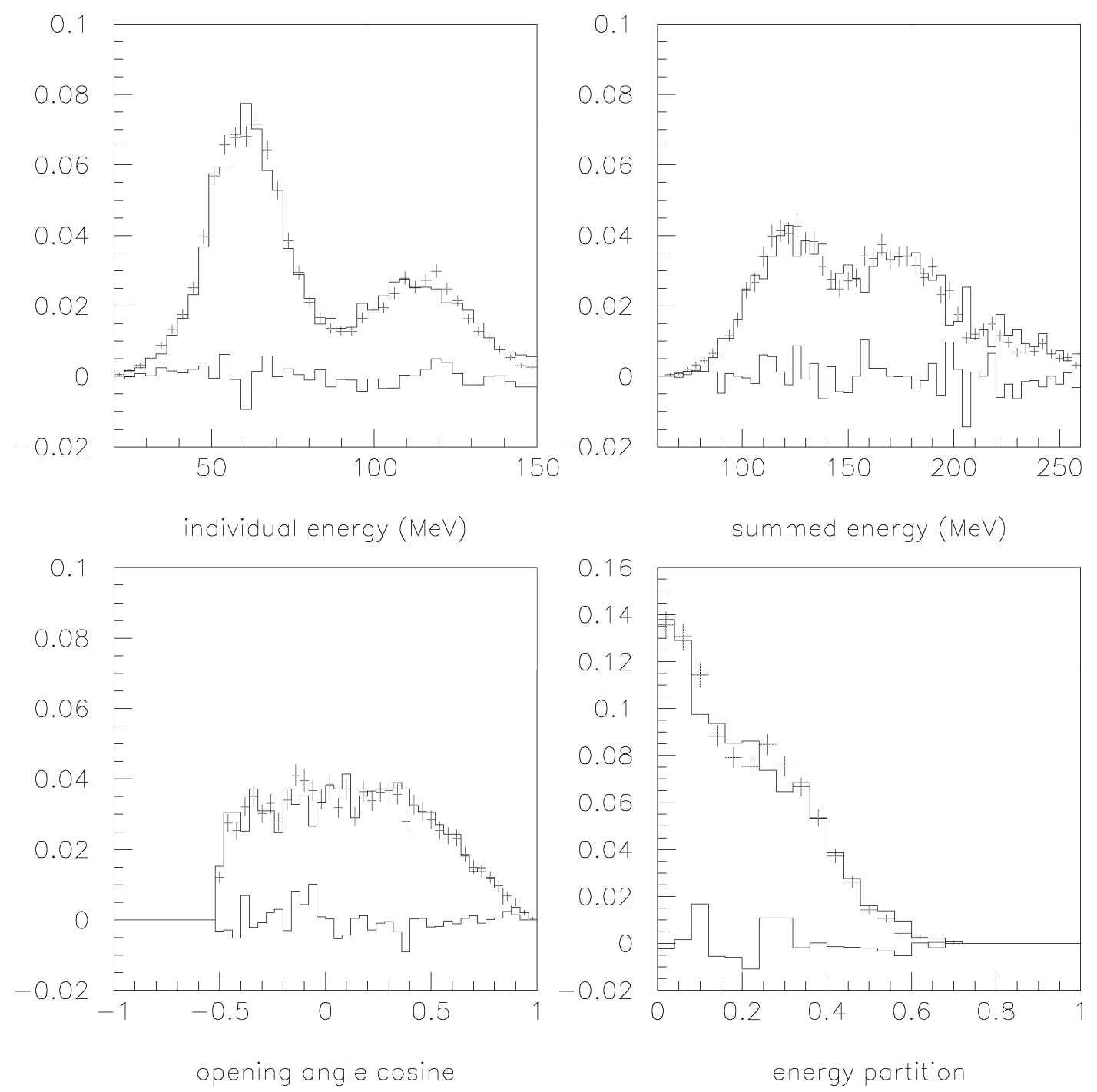

FIG. 11. Comparison of simulated data (solid line) and measured data (data points) for $\pi \pi$ background events. The plots are the individual photon energies (upper left), summed photon energies (upper right), opening angle (lower left) and energy partition (lower right). Also show is the difference between the simulated data and the measured data. 


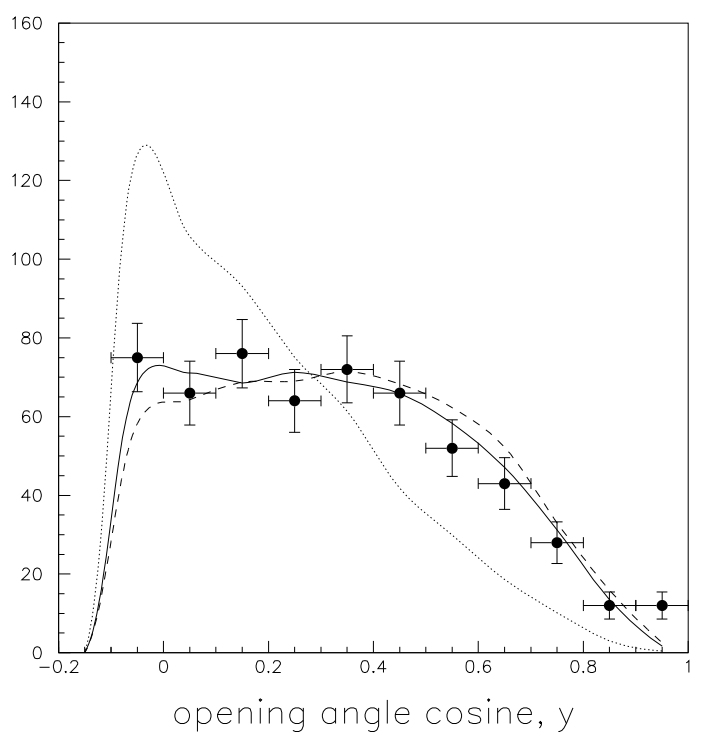

FIG. 12. Comparison of the opening angle distribution from the experimental data (closed circles) and the theoretical calculations (curves) for double radiative capture in pionic hydrogen. The solid curve is the full calculation, the dashed curve is the $\pi \pi$ term only, and the dotted curve is the NN term only. Note the theoretical curves have been normalized to the summed counts of the experimental spectrum for the comparison of the angular dependences.

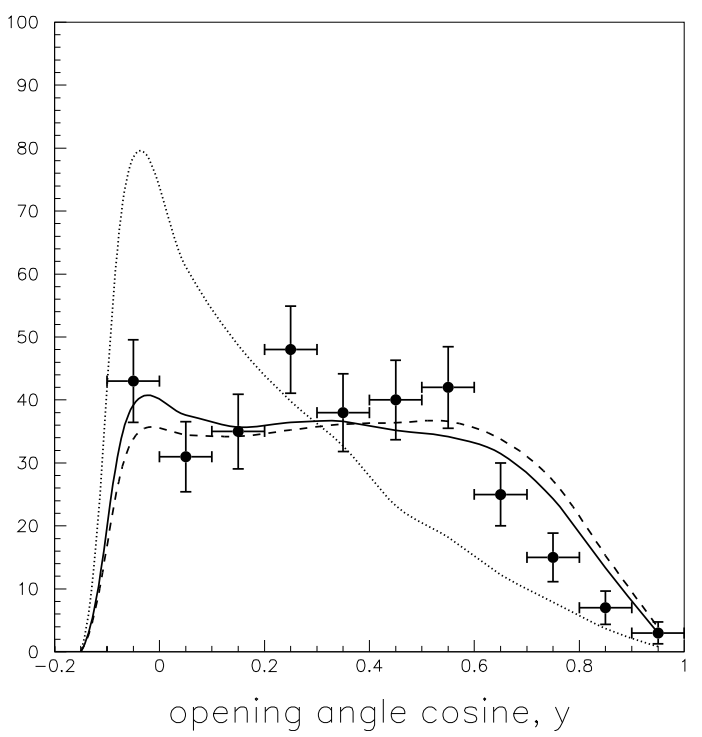

FIG. 13. Comparison of the opening angle distribution from the experimental data (closed circles) and the theoretical calculations (curves) for double radiative capture in pionic deuterium. The solid curve is the full calculation, the dashed curve is the $\pi \pi$ term only, and the dotted curve is the NN term only. Note the theoretical curves have been normalized to the summed counts of the experimental spectrum for the comparison of the angular dependences. 